\title{
Smallest Maximum Cable Tension Determination for Cable-Driven Parallel Robots
}

\author{
Hussein Hussein ${ }^{1}$, João Cavalcanti Santos ${ }^{1}$, Jean-Baptiste Izard $^{2}$, Marc Gouttefarde ${ }^{1}$
}

\begin{abstract}
The maximum cable tension is a crucial parameter in the design of a cable-driven parallel robot (CDPR) since the various mechanical components of the CDPR must be designed to safely withstand the loads induced by this maximum tension. For CDPRs having a number of cables at least equal to its number of DOFs, this paper deals with the determination of the smallest maximum cable tension vectors allowing a required wrench set to be feasible. The problem is formulated as the minimization of the maximum cable tension infinity norm under linear inequality constraints which include the wrench-feasibility constraints. The solution to this minimization problem is not unique, and the solution set is shown to be a convex polytope in the maximum tension space. Hence, various smallest maximum tension vectors generally exist and the computations of two different solution vectors are introduced. The first vector has all its components equal to the minimum infinity norm which can be directly obtained from the minimization problem inequality constraints. An algorithm is proposed to determine the second vector as the solution vector having the least possible value for each of its components. The computation of the smallest maximum tension vectors for general required wrench sets are then presented. The cases of particular wrench set definitions relevant to heavy payload manipulation applications are also introduced. Finally, these contributions are applied to the configuration (geometry) optimization of a large-dimension 6-DOF CDPR installed on a building facade to manipulate heavy payloads.
\end{abstract}

Index Terms-Cable-driven parallel robots (CDPR), parallel robots, design, wrench feasibility.

\section{INTRODUCTION}

$\mathbf{I}$ $\mathrm{N}$ an effort to introduce novel robotic systems in the construction industry, the motivation of this paper is the design of a cable-driven parallel robot (CDPR) intended for the construction or maintenance of building facades (Fig. 1). Tasks such as installation of curtain wall modules and facade cleaning may be efficiently performed using a mobile platform able to move in front of the building [1]. CDPRs have the capabilities to efficiently perform such tasks since all the degrees of freedom of their mobile platform can be controlled across a large workspace while handling heavy loads. CDPRs accomplishing tasks in a large workspace, possibly involving heavy payloads, have been demonstrated in several previous works [2], e.g., the Skycam [3], NIST RoboCrane [4], FAST radio telescope [5], CoGiRo [6], IPAnema [7], Fastkit [8], storage and retrieval machine [9], motion simulation platform [10], 3D printer [11].

1 Hussein Hussein, João Cavalcanti Santos, and Marc Gouttefarde are with LIRMM, Université de Montpellier, CNRS, Montpellier, France, \{hussein.hussein, joao.cavalcanti-santos, marc.gouttefarde\}elirmm. fr

2 Jean-Baptiste Izard is with Tecnalia Research \& Innovation, Basque Research Technology Alliance, Montpellier, France, jbi zardegmai l.com

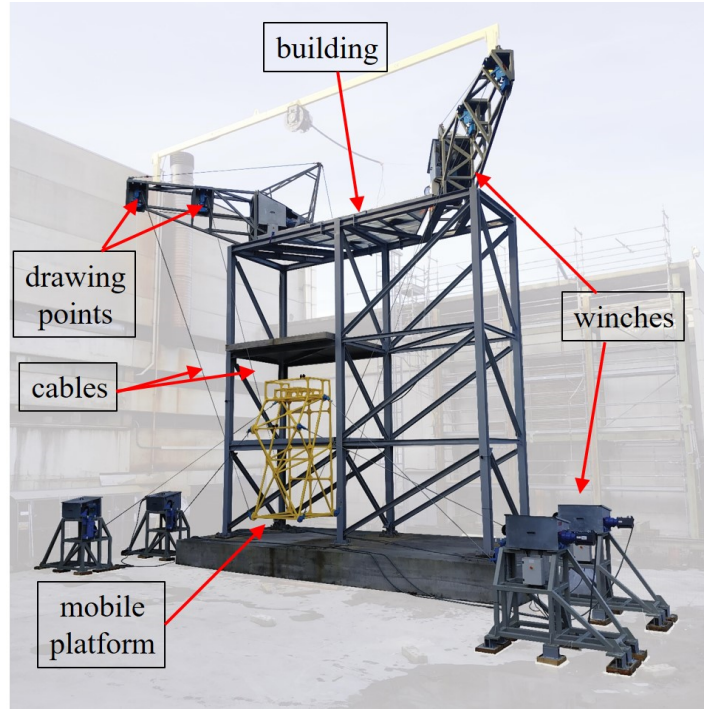

Fig. 1. CDPR prototype built in the framework of project Hephaestus [1].

Fundamental characteristics such as workspace size as well as wrench capabilities and stiffness across this workspace strongly depend on the CDPR configuration. The latter refers to the positions of both the cable exit points on the base frame and the attachment points on the mobile platform, as well as to the cable arrangement between these points. For instance, the size and shape of the wrench-feasible workspace are generally sensitive to changes in the dimensional parameters and in the cable arrangement of a CDPR [12], [13]. Finding an efficient CDPR configuration being given a set of requirements is generally challenging. The configuration can be synthesized based on previously known configurations or on the designer experience and intuition e.g., [9], [14], [15] but, in many previous works, the configuration is synthesized by formulating a constrained optimization problem.

The ability of the CDPR to generate wrenches in all possible directions despite the unidirectional character of cable actuation, i.e., wrench-closure [16]-[18], is included in the optimization problem in several of these previous works, e.g., [19]-[23]. The work presented in [19] is one of the first configuration design studies where wrench-closure but also cable collisions and multiple cable arrangement combinations were taken into account. In [21], [22], the problem of finding a CDPR configuration for which the Wrench-Closure Workspace (WCW) includes a prescribed workspace is tackled by means of convex relaxations allowing the formulation of a sufficient condition for a box to be fully inside the WCW. Besides, 
unilateral dexterity and force amplification quality indices have been proposed in [24] for $n$-degree-of-freedom (DOF) CDPRs driven by $m=n+1$ cables and recently generalized to the case $m>n+1$ in [25]. These indices, as well as others, e.g., [26]-[28], can be used in the formulation of a configuration design optimization problem to account for the unidirectional nature of cable actuation. Besides, the stiffness of CDPRs may be an issue and has thus been included into the formulation of configuration optimization problems in [28]-[30].

However, on the one hand, suspended CDPRs, e.g., [4][6], [31], cannot have wrench closure. On the other hand, in addition to the non-negative cable tension constraint, a maximum admissible cable tension $t_{\max }$ may have to be included in a CDPR configuration design problem to deal with possible overloads of mechanical components. Therefore, replacing or complementing wrench closure [32], wrenchfeasibility analysis, e.g., [12], [13], [33], [34] has been used to analyze CDPR wrench capabilities and to formulate configuration design optimization problems.

In the context of CDPR configuration design, minimum and maximum cable tensions $t_{\min }$ and $t_{\max }$ are considered in [27], where cable tension performance functions are defined. As a possible wrench-feasibility performance index, the socalled minimum degree of constraint satisfaction is proposed in [35], also known as the capacity margin [36]. Configuration optimization problems with the capacity margin as an objective function and constraints based on wrench feasibility are formulated in [36], [37] for usual CDPRs, and in [8] for a mobile CDPR where tipping constraints are also considered. Based on a wrench-feasibility analysis, the cable-suspended configuration of the CDPR CoGiRo is obtained by optimizing the maximum acceptable distance between the platform geometrical center and the center of mass [6]. If the designed robot is meant for a rather specific task, the configuration optimization can be based on the performance associated to this task. This design-to-task approach is considered for CDPRs in [38], where interval analysis is used to solve the corresponding constraint satisfaction problem, this approach being similar to the ones in [39], [40]. In [41], particle swarm optimization is used to optimize a cable-driven robot leg.

As proposed in our preliminary work [42], formulating a CDPR configuration optimization problem with the maximum cable tension $t_{\max }$ as the objective function to be minimized is relevant. Indeed, $t_{\max }$ is a crucial parameter since the various mechanical components of the CDPR must withstand this maximum cable tension safely. Consequently, the characteristics of these components strongly depend on the value of $t_{\max }$. In all the works cited above, the maximum cable tension $t_{\max }$ has a fixed value chosen from the outset of the design procedure. To the best of our knowledge, only two previous works [43], [44] do not consider a pre-selected constant $t_{\max }$. In [43], Dykstra's alternating projection algorithm is used to determine the minimum $t_{\max }$ allowing to balance a given wrench. This method may be used in a configuration design problem but Dykstra's algorithm is relatively slow. Moreover, in the framework of a design problem including wrenchfeasibility, all the individual wrenches (of a discretization) of the required wrench set must be considered. Hence, the use of this method can result in overly time-consuming computations since Dykstra's algorithm must, at each iteration of the design parameter optimization algorithm, be repeated for all the poses of a discretized prescribed workspace. Finding the smallest $t_{\max }$ such that all the wrenches of a given required wrench set are feasible without resorting to a brute-force wrench set discretization is thus relevant. In [44], an efficient means of calculating the smallest $t_{\max }$ is found in the form of a closedform expression. However, this closed-form expression of $t_{\max }$ is obtained by taking advantage of the particular form of the equations of a reduced-DOF CDPR performing translational motions and it cannot be applied to the general case of a $n$ DOF CDPR driven by $m$ cables $(m \geq n)$.

The main contribution of this paper is the determination of the smallest maximum cable tension vector $\mathbf{t}_{\max }$ such that all wrenches in a given required wrench set can be generated with feasible cable tensions $\mathbf{t}$, i.e., $\mathbf{t}_{\min } \leq \mathbf{t} \leq \mathbf{t}_{\max }$, where $\mathbf{t}_{\text {min }}$ is a given vector of minimum cable tensions. Among all the maximum cable tension vectors $\mathbf{t}_{\text {max }}$ allowing these wrenchfeasibility constraints to be satisfied, the smallest maximum cable tension vector is defined as the one having the smallest maximum component. The latter will be referred to as the smallest maximum tension value.

A preliminary version of this work was published in [42]. This preliminary work deals with the configuration design optimization of a CDPR while the present paper contribution is focused on the determination of the smallest maximum tension for $n$-DOF CDPRs driven by $m \geq n$ cables. More specifically, the novel contributions of the present paper are as follows.

First, in Section III, the case of a required wrench set reduced to a single wrench is addressed. The problem of determining the smallest maximum cable tension vector such that this wrench can be generated with feasible cable tensions is formulated as a minimization problem with linear inequality constraints. We prove that the smallest maximum tension value $t_{\max }^{*}$ can be calculated directly from the linear inequality constraints of this problem. For a pose inside the WCW, it is also shown that these inequality constraints are always consistent so that $t_{\max }^{*}$ always exists. Outside the WCW or for a non-existent WCW, conditions on the wrench and on $\mathbf{t}_{\min }$ for the constraints to be consistent, and thus for $t_{\max }^{*}$ to exist, are presented. Then, we point out that there exist infinitely many smallest maximum tension vectors allowing the wrench to be generated with feasible cable tensions In fact, we prove that the optimal solution set is a convex polytope. The determinations of two particular smallest maximum tension vectors are presented. The first one has all its components equal to $t_{\max }^{*}$ while the second one has the least possible value for each of its components. Subsequently, in Section IV, the determination of these smallest maximum cable tension vectors is extended to the case of a set of required wrenches. In particular, we show how the formula providing the smallest maximum tension value $t_{\max }^{*}$ can be modified to handle a wrench set instead of a single wrench. The computations involved in this formula for general wrench sets, including polytopes and ellipsoids, are presented. Moreover, for several practical wrench set definitions, closed-form expressions of wrench components permitting an efficient computation of $t_{\max }^{*}$ 


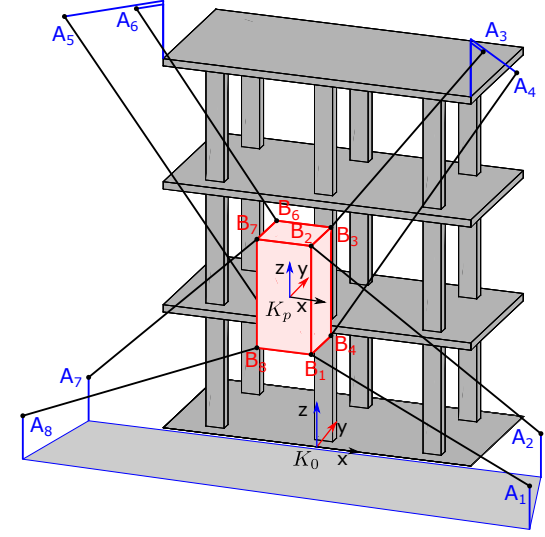

Fig. 2. Schematic representation of a CDPR installed on a building facade.

are introduced.

Finally, in Section V, these contributions are applied to the configuration design of a CDPR installed on the facade of a building to manipulate heavy loads. The previously defined practical wrench set definitions are used and the objective of the CDPR configuration design is to minimize the smallest maximum cable tension across a prescribed workspace. Based on the resulting best configuration, the 6-DOF large-dimension CDPR shown in Fig. 1 has been built and used for demonstrations of curtain wall module installation.

\section{BASIC MODELING AND WRENCH FeASIBILITY}

A schematic representation of a 6-DOF CDPR installed on a building facade is shown in Fig. 2. In the context of this work, the CDPR is intended to displace heavy payloads, at relatively low velocities and accelerations, and within a large workspace. Such heavy payload displacements are common in applications in the construction, lifting and other heavy industries. In such applications, low velocities are usually required for safety reasons. Consequently, this work focuses on CDPRs expected to work in a quasi-static manner and whose dynamics are neglected. The cable mass, and thus cable sagging, is also neglected. The latter assumption is reasonable in a design procedure where a very accurate model is not required contrary to the case of control and calibration. The cables are thus assumed to be straight line segments. As shown in Fig. 2, they are attached to the mobile platform at points $B_{i}$, referred to as the attachment points, and exit from the base at points $A_{i}$, referred to as the drawing points, where $i=1,2, \ldots, m$ and $m$ is the number of cables. $K_{0}$ is a fixed reference frame and $K_{p}$ is a frame attached to the CDPR mobile platform at its reference point $P$.

Based on these assumptions, the remainder of this section summarizes the wrench-feasibility analysis formalized in previous works on CDPRs, e.g., [12], [13], [33], [45].

\section{A. Wrench feasibility}

The wrench matrix $\mathbf{W}$ of a $n$-DOF CDPR driven by $m$ cables maps the cable tensions $\mathbf{t}$ to the wrench $\mathbf{f}$ applied by the cables on the mobile platform [46]:

$$
\mathbf{W t}=\mathbf{f} .
$$

The $n \times m$ wrench matrix $\mathbf{W}$ depends on the CDPR geometry (positions of points $A_{i}$ and $B_{i}$ ) and on the mobile platform pose. Its $i$-th column is denoted $\mathbf{w}_{i}\left(\mathbf{W}=\left[\mathbf{w}_{1}, \ldots, \mathbf{w}_{m}\right]\right)$. This paper deals with CDPRs with as many or more cables than DOFs, i.e., $m \geq n$.

A common necessary condition in the design of a CDPR is wrench feasibility. A given mobile platform pose is said to be wrench-feasible [12], [33], when a required wrench set RW is entirely contained within the available wrench set AW:

$$
\mathrm{RW} \subseteq \mathrm{AW} .
$$

On the one hand, the RW is the set of wrenches $\mathbf{f}$ that the cables must be able to generate at the mobile platform reference point $P$. Its exact definition depends on the task(s) to be accomplished by the CDPR. On the other hand, the AW is defined from admissible cable tensions:

$$
\mathrm{AW}=\left\{\mathbf{f}=\mathbf{W t} \mid \mathbf{t}_{\min } \leq \mathbf{t} \leq \mathbf{t}_{\max }\right\}
$$

where $\mathbf{t}_{\min }$ is the vector of minimum tensions, $\mathbf{t}_{\max }$ is the vector of maximum tensions, and $\leq$ denotes a componentwise inequality between two vectors. The latter notation is used in the remainder of the paper. The minimum and maximum admissible tensions may be defined differently for each cable, i.e., the components $t_{\min , i}$ (resp. $t_{\max , i}$ ) of $\mathbf{t}_{\min }$ (resp. $\mathbf{t}_{\max }$ ) are not necessarily all equal. Since cables can pull but not push, all components of $\mathbf{t}_{\min }$ are non-negative $\left(\mathbf{t}_{\min } \geq \mathbf{0}\right)$. In addition, to avoid cable sagging, strictly positive values are often used to define $\mathbf{t}_{\min }$. Besides, the components of $\mathbf{t}_{\max }$ are defined to account for the capabilities and limitations of the cables, winches, supports, etc.

\section{B. Wrench feasibility as a system of linear inequalities}

The feasibility conditions in (2) are necessary conditions for the CDPR to be able to produce all wrenches in the RW with admissible cable tensions. The set of admissible cable tensions is a hyperrectangle in tension space. According to (3), the AW is the image of this hyperrectangle under the linear map represented by matrix $\mathbf{W}$. The AW is then a convex polytope in the wrench space (more precisely, it is a zonotope [33]) and it can thus be represented as the solution set of a system of linear inequalities [47]:

$$
\mathrm{AW}=\{\mathbf{f} \mid \mathbf{C f} \leq \mathbf{d}\}
$$

This system of linear inequalities is defined by the $p \times n$ matrix $\mathbf{C}$ and $p$-dimensional column vector $\mathbf{d}$. Each inequality in this system defines a facet of the AW. $\mathbf{C}$ and $\mathbf{d}$ can be determined by means of the hyperplane shifting method introduced in [33]. In brief, each combination $\left\{\mathbf{w}_{i_{1}}, \mathbf{w}_{i_{2}}, \ldots, \mathbf{w}_{i_{n-1}}\right\}$ of $n-1$ linearly independent columns of the $n \times m$ wrench matrix W spans a facet-defining hyperplane of the AW and thus provides two rows of $\mathbf{C}$, say $\mathbf{c}_{k}$ and $\mathbf{c}_{l}$. Each of these two row vectors $\mathbf{c}_{k}$ and $\mathbf{c}_{l}$ spans the one-dimensional nullspace of the matrix $\mathbf{M}_{I}=\left[\mathbf{w}_{i_{1}}, \mathbf{w}_{i_{2}}, \ldots, \mathbf{w}_{i_{n-1}}\right]^{T}$, where $I$ denotes the index set $I=\left\{i_{1}, \ldots, i_{n-1}\right\} \subset\{1, \ldots, m\}$. Moreover, $\mathbf{c}_{k}$ and $\mathbf{c}_{l}$ are pointing in opposite directions, i.e.:

$$
\mathbf{c}_{k}=-\mathbf{c}_{l}=\operatorname{null}\left(\mathbf{M}_{l}\right)^{T} .
$$


In other words, $\mathbf{c}_{k}$ and $\mathbf{c}_{l}$ are orthogonal to the $n-1$ column vectors $\mathbf{w}_{i_{1}}, \mathbf{w}_{i_{2}}, \ldots, \mathbf{w}_{i_{n-1}}$. Hence, the number $p$ of rows of $\mathbf{C}$ and $\mathbf{d}$ is less or equal to $2 C_{m}^{n-1}$ where $C_{m}^{n-1}$ is the number of $(n-1)$-combinations of the $m$ columns of $\mathbf{W}$. Note that $p$ is equal to $2 C_{m}^{n-1}$ when each $(n-1)$-combination of columns of $\mathbf{W}$ is a set of linearly independent vectors. Provided that $\mathbf{t}_{\text {max }} \geq \mathbf{t}_{\text {min }}$, the component $d_{j}$ of $\mathbf{d}$ corresponding to the row $\mathbf{c}_{j}$ of $\mathbf{C}$ is given by [45]:

$$
d_{j}=\sum_{i \in I_{j}^{+}} t_{\max , i} \mathbf{c}_{j} \mathbf{w}_{i}+\sum_{i \in I_{j}^{-}} t_{\min , i} \mathbf{c}_{j} \mathbf{w}_{i}
$$

where $I_{j}^{+}$and $I_{j}^{-}$are the subsets of $\{1, \ldots, m\}$ defined as $I_{j}^{+}=\left\{i \mid \mathbf{c}_{j} \mathbf{w}_{i}>0\right\}$ and $I_{j}^{-}=\left\{i \mid \mathbf{c}_{j} \mathbf{w}_{i}<0\right\}$, and $t_{\max , i}$ and $t_{\min , i}$ are the $i$-th components of $\mathbf{t}_{\max }$ and $\mathbf{t}_{\min }$, respectively.

Taking advantage of the formulation of the AW as a system of linear inequalities in (4), the wrench-feasibility conditions in (2), i.e., $\mathrm{RW} \subseteq \mathrm{AW}$, are equivalent to:

$$
\mathbf{C f} \leq \mathbf{d}, \forall \mathbf{f} \in \mathrm{RW} .
$$

The wrench-feasibility conditions formulated in (7) can generally be tested in a straightforward manner [33] and can also be used to conduct particular wrench-feasibility analyses, e.g., [6], [8]. In the present paper, they will be used to determine the smallest maximum tension vectors verifying the wrenchfeasibility constraints (2).

\section{Smallest Maximum Cable Tension}

This section introduces an original formulation of the smallest maximum cable tension such that a wrench $\mathbf{f}$ belongs to the available wrench set AW, i.e., such that $\mathbf{f}$ can be produced at the CDPR mobile platform with admissible cable tensions.

\section{A. Minimization problem formulation and optimal solution}

Being given a vector $\mathbf{t}_{\min }$ of minimum tensions and a wrench $\mathbf{f}$, if $\mathbf{C f} \leq \mathbf{d}$ in (4), $\mathbf{t}_{\max }$ is such that $\mathbf{f}$ belongs to the available wrench set AW. With the definition of $d_{j}$ in (6), each row of $\mathbf{C f} \leq \mathbf{d}$ can be written in terms of the components $t_{\max , i}$ of $\mathbf{t}_{\max }$ :

$$
\sum_{i \in I_{j}^{+}} \mathbf{c}_{j} \mathbf{w}_{i} t_{\max , i} \geq \mathbf{c}_{j} \mathbf{f}-\sum_{i \in I_{j}^{-}} t_{\min , i} \mathbf{c}_{j} \mathbf{w}_{i} .
$$

The components of $\mathbf{t}_{\max }$ must satisfy (8) for all $j, 1 \leq j \leq p$, for $\mathbf{f}$ to belong to the AW. Moreover, it is important to note that (6) is obtained by assuming that $\mathbf{t}_{\max } \geq \mathbf{t}_{\min }$ [45]. Hence, the inequalities $\mathbf{t}_{\max } \geq \mathbf{t}_{\min }$ have to be enforced since otherwise there may exist some vectors $\mathbf{t}_{\max }$ satisfying (8) but having some components $t_{\max , i}$ smaller than $t_{\min , i}$, An example of such a vector will be given in Section III-D.

The inequalities (8) (for all $j$ ) and $\mathbf{t}_{\max } \geq \mathbf{t}_{\min }$ can be aggregated into a single system of linear inequalities:

$$
\mathbf{A} \mathbf{t}_{\max } \geq \mathbf{b}
$$

which represents the set of vectors $\mathbf{t}_{\max }$ such that $\mathbf{f}$ belongs to the AW. The $q \times m$ matrix $\mathbf{A}$ and $q \times 1$ vector $\mathbf{b}$ in this system of linear inequalities, where $q=p+m$, can be written:

$$
\mathbf{A}=\left[\begin{array}{c}
\mathbf{A}_{1} \\
\mathbf{A}_{2}
\end{array}\right]=\left[\begin{array}{c}
\mathbf{a}_{1} \\
\vdots \\
\mathbf{a}_{q}
\end{array}\right], \quad \mathbf{b}=\left[\begin{array}{c}
\mathbf{b}_{1} \\
\mathbf{b}_{2}
\end{array}\right]=\left[\begin{array}{c}
b_{1} \\
\vdots \\
b_{q}
\end{array}\right]
$$

where the components of matrix $\mathbf{A}_{1}$ and vector $\mathbf{b}_{1}$ are defined as follows, for $j=1$ to $p$ :

$$
\begin{gathered}
a_{j i}=\mathbf{c}_{j} \mathbf{w}_{i}, \text { for } i \in I_{j}^{+} \quad \text { and } \quad a_{j i}=0, \text { for } i \notin I_{j}^{+} \\
b_{j}=\mathbf{c}_{j} \mathbf{f}-\sum_{i \in I_{j}^{-}} t_{\min , i} \mathbf{c}_{j} \mathbf{w}_{i}
\end{gathered}
$$

and $\quad \mathbf{A}_{2}=\left[\mathbf{a}_{p+1}^{T}, \ldots, \mathbf{a}_{q}^{T}\right]^{T}=\mathbf{I}_{n}$ (identitity matrix) and $\mathbf{b}_{2}=\left[b_{p+1}^{T}, \ldots, b_{q}^{T}\right]^{T}=\mathbf{t}_{\text {min }}$. With these definitions, the first $p$ rows of (9) are equivalent to $\mathbf{C f} \leq \mathbf{d}$ and its last $m$ rows are $\mathbf{t}_{\max } \geq \mathbf{t}_{\min }$. Note that $a_{j i} \geq 0 \forall j, i$ and that each row of $\mathbf{A}$ contains at least $n-1$ zeros since $\mathbf{c}_{j}$ is orthogonal to $n-1$ wrenches $\mathbf{w}_{i}$ according to (5).

For given minimum cable tensions $\mathbf{t}_{\text {min }}$, mobile platform pose, and wrench $\mathbf{f}$, the matrix $\mathbf{A}$ and vector $\mathbf{b}$ are constant. The problem of determining the smallest maximum cable tension vectors such that $\mathbf{f} \in \mathrm{AW}$ can then be formulated as the following optimization problem:

$$
\begin{gathered}
\min _{\mathbf{t}_{\max }} \max _{i} t_{\max , i} \\
\text { subject to } \quad \mathbf{A t}_{\max } \geq \mathbf{b}
\end{gathered}
$$

where $\mathbf{A}$ and $\mathbf{b}$ are defined in (10). Note that $\max t_{\max , i}=$ $\left\|\mathbf{t}_{\max }\right\|_{\infty}$ where $\|\cdot\|_{\infty}$ is the infinity norm.

Since $a_{j i} \geq 0 \forall j, i$, it suffices to take a $\mathbf{t}_{\max }$ with sufficiently large components to have $\mathbf{A} \mathbf{t}_{\max } \geq \mathbf{b}$ (see Section III-B for a discussion of the case $\mathbf{a}_{j}=\mathbf{0}$ ). Moreover, considering only one inequality (one row) of (9), $a_{j i} \geq 0 \forall i$ also implies that the smallest $\mathbf{t}_{\max }$ (in the infinity norm sense) such that $\mathbf{a}_{j} \mathbf{t}_{\max } \geq b_{j}$ is obtained by setting this inequality to equality. Then, considering a component of $\mathbf{t}_{\max }$ multiplied by a strictly positive $a_{j i}$, reducing the value of this component leads necessarily to increasing the value of another component of $\mathbf{t}_{\max }$ in order to maintain this equality. Consequently, the smallest $\mathbf{t}_{\max }$ such that $\mathbf{a}_{j} \mathbf{t}_{\max } \geq b_{j}$ can have all its components equal. This property can also be seen from simple $2 \mathrm{D}$ or $3 \mathrm{D}$ drawings ${ }^{1}$ and it leads to the following optimal solution to (13):

$$
\mathbf{t}_{\max }^{*}=\max _{j \in J_{+}}\left(\frac{b_{j}}{\sum_{i=1}^{m} a_{j i}}\right) \mathbf{1}_{m}
$$

where $\mathbf{1}_{m}=[1,1, \ldots, 1]^{T} \in \mathbb{R}^{m}$ and $J_{+}=\left\{j \mid \sum_{i=1}^{m} a_{j i}>0\right\}$ or equivalently $J_{+}=\left\{j, 1 \leq j \leq p \mid I_{j}^{+} \neq \emptyset\right\} \cup\{p+1, \ldots, q\}$. The fact that $\mathbf{t}_{\max }^{*}$ is an optimal solution to (13) is formally proved in Appendix A.

The following properties of $\mathbf{t}_{\max }^{*}$ and (14) are pointed out.

- All the components of $\mathbf{t}_{\max }^{*}$ in (14) are equal and:

$$
\left\|\mathbf{t}_{\max }^{*}\right\|_{\infty}=t_{\max }^{*}=\max _{j \in J_{+}}\left(\frac{b_{j}}{\sum_{i=1}^{m} a_{j i}}\right)=\max _{j \in J_{+}}\left(\frac{b_{j}}{\sum_{a_{j i}>0} a_{j i}}\right) \text {. }
$$

In terms of $\mathbf{f}$ and $\mathbf{t}_{\min }$, we have:

$$
\begin{cases}t_{\max }^{*}=\frac{\mathbf{c}_{h} \mathbf{f}-\sum_{i \in I_{h}^{-}} t_{\min , i} \mathbf{c}_{h} \mathbf{w}_{i}}{\sum_{i \in I_{h}^{+}} \mathbf{c}_{h} \mathbf{w}_{i}} & \text { when } h \leq p \\ t_{\max }^{*}=t_{\min , h} & \text { when } h>p\end{cases}
$$

\footnotetext{
${ }^{1}$ The level sets of the infinity norm $\left\|\mathbf{t}_{\max }\right\|_{\infty}$ are squares in $2 \mathrm{D}$ (resp. cubes in 3D) and the smallest level set such that $\mathbf{a}_{j} \mathbf{t}_{\max } \geq b_{j}$ has one of its vertices touching the line $\mathbf{a}_{j} \mathbf{t}_{\max }=b_{j}$ (resp. plane). This vertex corresponds to a $\mathbf{t}_{\max }$ having all its components equal.
} 
where $h$ is the index of the row of (9) defined as:

$$
h=\underset{j \in J_{+}}{\operatorname{argmax}}\left(\frac{b_{j}}{\sum_{i=1}^{m} a_{j i}}\right) .
$$

- By definition of the last $m$ rows of $\mathbf{A}$ and last $m$ components of $\mathbf{b}$, we have $\mathbf{t}_{\max }^{*} \geq \mathbf{t}_{\min }(\geq \mathbf{0})$.

- The index set $J_{+}$is equal to $\{1,2, \ldots, q\}$ if and only if the pose of the mobile platform belongs to the WCW, since the latter is a necessary and sufficient condition for $I_{j}^{+} \neq \emptyset$ for all $j, 1 \leq j \leq p$ (see Section III-B).

- When $\operatorname{rank}(\mathbf{W})=n$, it is proved in Appendix A that $\bigcup_{j} I_{j}^{+}=\{1, \ldots, m\}$ so that all components of $\mathbf{t}_{\max }$ are impacted by the wrench feasibility conditions on $\mathbf{f}$ (first $p$ rows of $\mathbf{A t}_{\max } \geq \mathbf{b}$ ).

The system of linear inequalities $\mathbf{A t}_{\max } \geq \mathbf{b}$ should be consistent for $\mathbf{t}_{\max }^{*}$ in (14) to be well defined (cf. Appendix A). The conditions under which this system of linear inequalities possesses some solutions $\mathbf{t}_{\max }$ is examined in the next section.

\section{B. Consistency of the inequality constraints}

By definition, $\mathbf{c}_{j}$ is orthogonal to $n-1$ columns of $\mathbf{W}$ (Eq. (5)). Hence, in each row of $\mathbf{A}$, at least $n-1$ components $a_{j i}$ are equal to zero. Moreover, for $j=1$ to $p$, a row $j$ of $\mathbf{A}$ is the zero vector $\left(\mathbf{a}_{j}=\mathbf{0}\right.$ and $\left.j \notin J_{+}\right)$if and only if $I_{j}^{+}=\emptyset$ (for $j=p+1$ to $q$, by definition, $\mathbf{a}_{j} \neq \mathbf{0}$ ). Consequently, when $I_{j}^{+}=\emptyset$, no component of $\mathbf{t}_{\max }$ is involved in the $j$-th row of the feasibility conditions (9) and some components of $\mathbf{t}_{\min }$ must satisfy the following inequality:

$$
\mathbf{c}_{j} \mathbf{f} \leq \sum_{i \in I_{j}^{-}} t_{\min , i} \mathbf{c}_{j} \mathbf{w}_{i} \quad \Longleftrightarrow \quad b_{j} \leq 0 .
$$

If (18) is not satisfied, $b_{j}>0$ and, since $\mathbf{a}_{j}=\mathbf{0}$, the system of inequalities (9) is inconsistent which means that $\nexists \mathbf{t}_{\text {max }} \in \mathbb{R}^{m} \mid \mathbf{A t}_{\text {max }} \geq \mathbf{b}$. It is proved in Appendix $\mathrm{B}$ that $I_{j}^{+} \neq \emptyset \forall j$ if and only if the pose of the CDPR mobile platform belongs to the wrench-closure workspace (WCW). Hence, inside the WCW, any wrench can be generated with nonnegative cable tensions and no condition like the one in (18) needs to be verified to ensure the existence of a $\mathbf{t}_{\max }$ such that $\mathbf{f}$ belongs to the AW. However, outside the WCW or for a nonexistent $\mathrm{WCW}$, the inequalities (18) for all $j$ such that $I_{j}^{+}=\emptyset$ must be fulfilled for $\mathbf{f}$ to be feasible at a given platform pose, i.e., for being able to find a $\mathbf{t}_{\max }$ such that $\mathbf{f}$ can be generated with cable tensions $\mathbf{t}$ satisfying $\mathbf{t}_{\min } \leq \mathbf{t} \leq \mathbf{t}_{\max }$. Indeed, the inequalities (18) ensure that all rows $j$ of $\mathbf{A t}_{\max } \geq \mathbf{b}$ such that $\mathbf{a}_{j}=\mathbf{0}$ are satisfied and, regarding the other rows, since $a_{j i} \geq 0 \forall j, i$, one can always find a $\mathbf{t}_{\max }$ verifying $\mathbf{A t}_{\max } \geq \mathbf{b}$ (it suffices to take a $\mathbf{t}_{\max }$ with sufficiently large components).

Note that the WCW of a suspended CDPR (such as the one in [6], [48]) is nonexistent so that there exist indices $j$ such that $I_{j}^{+}=\emptyset$ for all poses of the mobile platform of this type of CDPR.

\section{Set of optimal solutions: A convex polytope}

The smallest maximum cable tension vector given in (14) is not the unique optimal solution to (13). Indeed, it is obtained

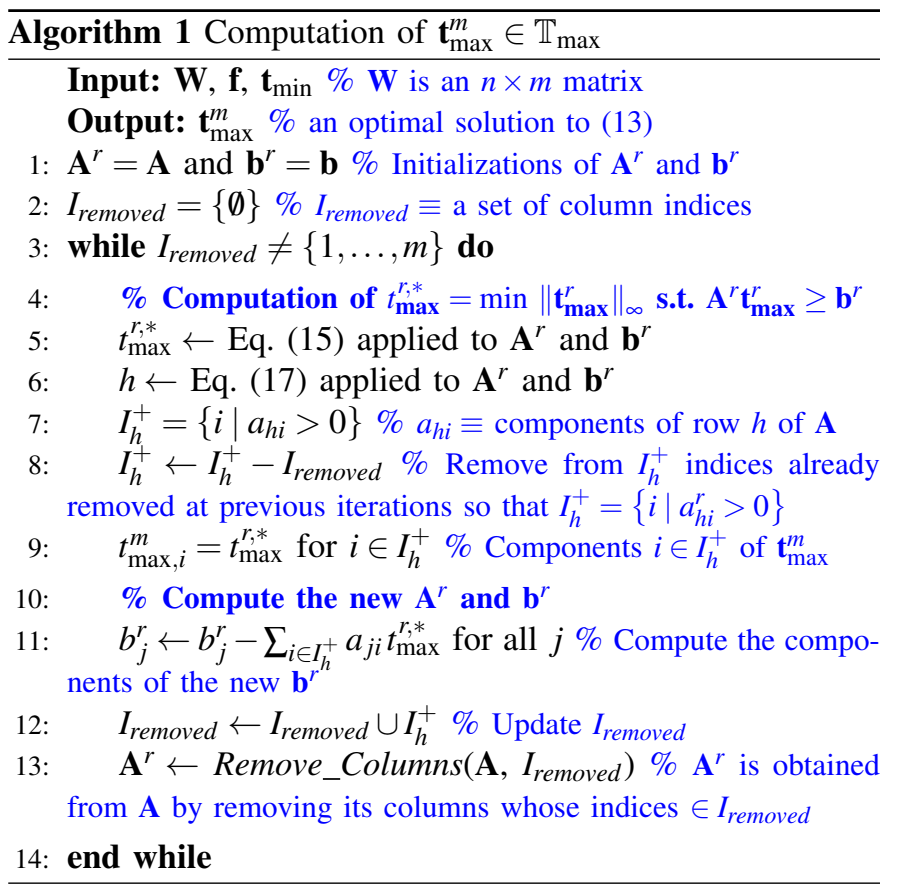

from row $h$ of (9), with $h$ defined in (17), and the components $t_{\mathrm{max}, i}$ of $\mathbf{t}_{\mathrm{max}}$ such that $i \notin I_{h}^{+}$are not involved in this row since $a_{h i}=0$ for $i \notin I_{h}^{+}$. Other optimal solutions to (13) may thus be obtained since the maximum cable tension components $t_{\mathrm{max}, i}, i \notin I_{h}^{+}$, can be smaller than the optimal value $t_{\max }^{*}$ defined in (15) while the inequality constraints in (9) remain satisfied.

In fact, for a given pose of the CDPR mobile platform and given minimum tensions $\mathbf{t}_{\min }$, let $\mathbb{T}_{\max }$ be the set of all optimal solutions to (13). In other words, $\mathbb{T}_{\max }$ is the set of smallest maximum cable tension vectors $\mathbf{t}_{\max }$ such that f can be generated with cable tensions $\mathbf{t}$ verifying (3). It is proved in Appendix $\mathrm{C}$ that $\mathbb{T}_{\max }$ is a convex polytope whose representation as the solution set of a system of linear inequalities can directly be obtained from (9) and (14)-(15).

Based on this representation, it can be of interest to find maximum cable tension vectors in $\mathbb{T}_{\max }$ other than $\mathbf{t}_{\max }^{*}$. Indeed, referring to Appendix $\mathrm{C}$ and in particular to (55) and (56), any such maximum cable tension vector has several (but not all) components smaller than $t_{\max }^{*}$. In order to compute these other maximum cable tension vectors, a vertex enumeration algorithm [47], [49], may be used to find all the vertices of $\mathbb{T}_{\max }$. However, such calculations require non-negligible computation times and difficult issues linked to degeneracy may have to be dealt with because the rows of matrix $\mathbf{A}$ contain at least $n-1$ zeros ( $\mathbf{c}_{j}$ is orthogonal to $n-1$ wrenches $\mathbf{w}_{i}$ ).

As an alternative, an efficient and original method allowing the computation of a maximum cable tension vector in $\mathbb{T}_{\max }$ is proposed. This maximum cable tension vector is denoted $\mathbf{t}_{\max }^{m}$ and its components can be significantly smaller than $t_{\max }^{*}$ (cf the examples in Section III-D). The method to compute $\mathbf{t}_{\max }^{m}$ consists in solving the minimization problem (13) several times in order to successively minimize all the components of $\mathbf{t}_{\max }$. Thereby, $\mathbf{t}_{\max }^{m}$ is the solution vector in $\mathbb{T}_{\max }$ having the least possible value for each of its components. Specifically, as noted at the beginning of this section and according to 


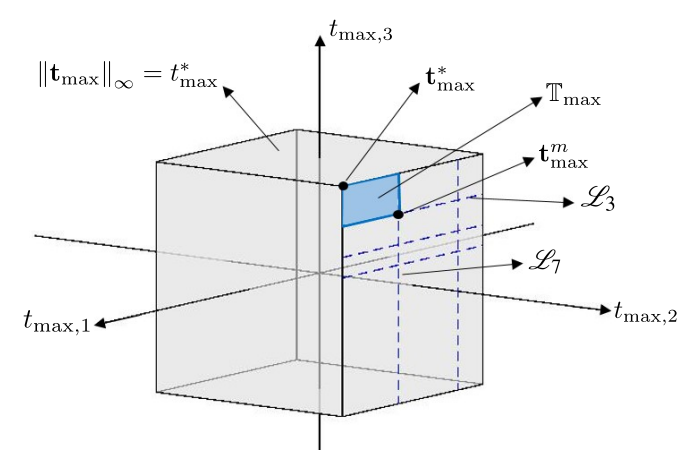

Fig. 3. Set $\mathbb{T}_{\max }$ and vectors $\mathbf{t}_{\max }^{*}$ and $\mathbf{t}_{\max }^{m}$ shown in the 3D space of the maximum tension vectors $\mathbf{t}_{\max }$ for the planar 2-DOF 3-cable CDPR example at position $P=(0.3 ; 1)$ - In this example, $h$ defined in (17) is equal to 5 . Hence, according to (20), we have $t_{\max }^{*}=b_{5} / a_{52}=490.24 \mathrm{~N}$ and $\mathbb{T}_{\max }$ lies on the facet $t_{\max , 2}=490.24 \mathrm{~N}$ of the infinity norm level set. This level set is the set of $\mathbf{t}_{\max }$ such that $\left\|\mathbf{t}_{\max }\right\|_{\infty}=t_{\max }^{*}$ and it corresponds to the facets of the grey cube shown in the figure. Referring to the constraints of the minimization problem (13), the straight line segments $\mathscr{L}_{3}$ and $\mathscr{L}_{7}$ are the intersections of the hyperplanes $\mathbf{a}_{3} \mathbf{t}_{\max }=b_{3}$ and $\mathbf{a}_{7} \mathbf{t}_{\max }=b_{7}$ with this cube, respectively. As can be seen, $\mathscr{L}_{3}$ and $\mathscr{L}_{7}$ delimit boundaries of $\mathbb{T}_{\max }$ and $\mathbf{t}_{\max }^{m}$ is the smallest maximum tension in $\mathbb{T}_{\max }$.

(55)-(57), there exists $\mathbf{t}_{\max } \in \mathbb{T}_{\max }$ whose components of index $i \notin I_{h}^{+}$are smaller than $t_{\max }^{*}$. These components can then in turn be minimized by suitably formulating and solving successive minimization problems similar to (13) as detailed in Appendix D and illustrated by the implementation shown in Algorithm 1. Finally, as proved in Appendix E, note that:

$$
\left\{\mathbf{t}_{\max } \mid t_{\max , i}^{m} \leq t_{\max , i} \leq t_{\max }^{*}, i=1 \ldots m\right\} \subseteq \mathbb{T}_{\max }
$$

i.e., the box defined in the left-hand side of (19) is fully contained in $\mathbb{T}_{\max }{ }^{2}$

\section{Examples}

As a first example, let us consider a simple planar twoDOF CDPR driven by three cables with a mobile platform reduced to a point mass. The cable drawing point coordinates in the fixed reference frame are $A_{1}=(0 ; 0), A_{2}=(1 ; 2)$ and $A_{3}=(-1 ; 2)$. The wrench applied by the cables is $\mathbf{f}=\left[f_{x}, f_{z}\right]^{T} \in \mathbb{R}^{2}$ and the cable tensions are $\mathbf{t}=\left[t_{1}, t_{2}, t_{3}\right]^{T}$. The minimum tension $t_{\min }$ for each cable is $100 \mathrm{~N}$.

The point mass position $P=(0.3 ; 1)$ is considered. At this position, the point mass is fully constrained by the cables. As explained in Section II-B, the rows $\mathbf{c}_{j}$ of the $6 \times 2$ matrix $\mathbf{C}$ involved in (4) are computed ( $p=6$ and $n=2)$. Then, according to their definitions in Section III-A, the $9 \times 3$ matrix $\mathbf{A}$ and vector $\mathbf{b}(q=9)$ are obtained as:

$$
\mathbf{A}=\left[\begin{array}{ccc}
0 & 0 & 0.934 \\
0 & 0.314 & 0 \\
0 & 0 & 0.999 \\
0.314 & 0 & 0 \\
0 & 0.999 & 0 \\
0.934 & 0 & 0 \\
1.000 & 0 & 0 \\
0 & 1.000 & 0 \\
0 & 0 & 1.000
\end{array}\right] \quad \mathbf{b}=\left[\begin{array}{c}
175.0612 \\
-50.23 \\
318.12 \\
-186.83 \\
489.75 \\
-296.41 \\
100.00 \\
100.00 \\
100.00
\end{array}\right]
$$

${ }^{2}$ We never found vectors of $\mathbb{T}_{\max }$ outside of this box but we do not have a proof of $\mathbb{T}_{\max }$ being equal to this box. where the wrench $\mathbf{f}=[0,500]^{T}(\mathrm{~N})$ has been considered to compute $\mathbf{b}$. A and $\mathbf{b}$ define the inequality constraints of (13) and the optimal vectors $\mathbf{t}_{\max }^{*}$ and $\mathbf{t}_{\max }^{m}$ are calculated as:

$$
\mathbf{t}_{\max }^{*}=\left[\begin{array}{l}
490.24 \\
490.24 \\
490.24
\end{array}\right](\mathrm{N}) \quad \mathbf{t}_{\max }^{m}=\left[\begin{array}{l}
100.00 \\
490.24 \\
318.44
\end{array}\right](\mathrm{N}) .
$$

These optimal vectors and the set $\mathbb{T}_{\max }$ of all optimal solutions to (13) are shown in Fig. 3.

The second example is a 6-DOF 8-cable fully-constrained CDPR (configuration 23 in Fig. 13) for which the matrices $\mathbf{C}$ and $\mathbf{A}$ have dimensions $112 \times 6$ and $120 \times 8$, respectively. An example of smallest maximum tension vectors obtained for this CDPR configuration are (in N):

$$
\mathbf{t}_{\max }^{*}=\left[\begin{array}{c}
4656 \\
4656 \\
4656 \\
4656 \\
4656 \\
4656 \\
4656 \\
4656
\end{array}\right] \quad \mathbf{t}_{\max }^{m}=\left[\begin{array}{c}
200 \\
3783 \\
226 \\
200 \\
1075 \\
1702 \\
4656 \\
767
\end{array}\right]
$$

where $t_{\min }=200 \mathrm{~N}$ in this example. It can be noticed that some maximum tension values in $\mathbf{t}_{\max }^{m}$ are significantly smaller than those in $\mathbf{t}_{\max }^{*}$ and that some components of $\mathbf{t}_{\max }^{m}$ are equal to $t_{\min }$. In fact, if the inequalities $\mathbf{t}_{\max } \geq \mathbf{t}_{\min }$ were not enforced by being included in (9), the fourth component of $\mathbf{t}_{\max }^{m}$ in (22) would have been smaller than $t_{\min }=200 \mathrm{~N}$. This result can be verified by removing the last $m$ rows of (9) and then applying Algorithm 1.

\section{Smallest Maximum Cable Tension for a Set of WRENCHES}

In Section III, the determination of the smallest $\mathbf{t}_{\max }$ such that a given wrench $\mathbf{f}$ can be generated with admissible cable tensions (i.e., tensions verifying (3)) has been introduced. In the present section, this contribution is extended to the case of a set of wrenches. Moreover, for several practical wrench set definitions, closed-form expressions of the components of wrenches permitting an efficient computation of the smallest maximum tension vectors are given.

\section{A. Problem formulation and solution}

Referring to Section II-A, a common necessary condition in the design of a CDPR is to satisfy wrench-feasibility conditions formulated as in (2). For a given pose of the CDPR mobile platform, determining the smallest maximum cable tension vectors such that (2) is satisfied can be formulated as the following optimization problem:

$$
\min _{\mathbf{t}_{\max }} \max _{i} t_{\max , i}
$$

subject to $\quad \mathbf{A t}_{\max } \geq \mathbf{b}, \forall \mathbf{f} \in \mathrm{RW}$

where RW is the required wrench set defined in Section II-A, and $\mathbf{A}$ and $\mathbf{b}$ are defined in (10). Note that $\mathbf{b}$ depends on the wrench $\mathbf{f}$ according to the definitions of its components $b_{j}$ 


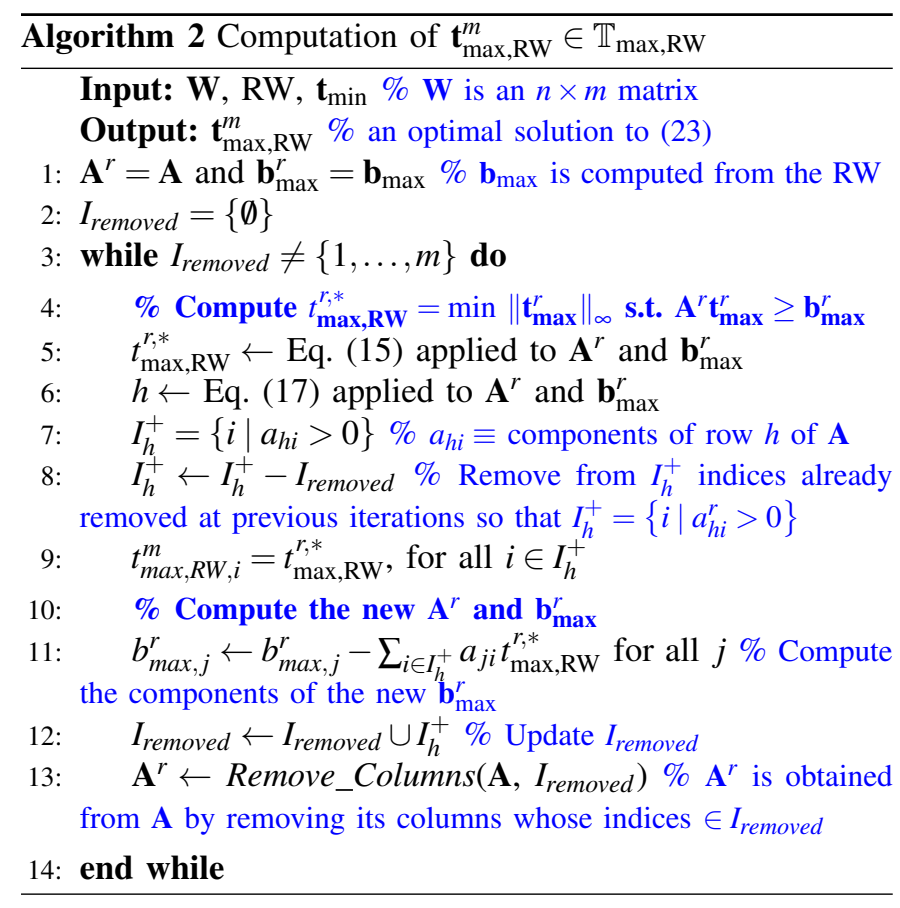

in (12) and that $\mathbf{A}$ is independent of the wrench $\mathbf{f}$ according to (11). A depends only on the wrench matrix $\mathbf{W}$ and is thus constant for a given pose of the CDPR.

The minimization problem in (23) is an extension of (13) to the case of a set of wrenches RW. As detailed in Section III-C, there exists a set of optimal solutions $\mathbb{T}_{\max }$ to the minimization problem (13) and the computations of two particular optimal solutions $\mathbf{t}_{\max }^{*}$ and $\mathbf{t}_{\max }^{m}$ have been introduced. The infinity norms of these optimal solutions are all equal to $t_{\text {max }}^{*}$ defined in (15). Examining (15) and since the $a_{j i}$ are independent of $\mathbf{f}$, it can be inferred that the optimal value of (23) is:

$$
t_{\max , \mathrm{RW}}^{*}=\max _{j \in J_{+}}\left(\frac{\max _{\mathbf{f} \in \mathrm{RW}} b_{j}}{\sum_{i=1}^{m} a_{j i}}\right)
$$

and thus that an optimal solution to (23) is the vector $\mathbf{t}_{\text {max, } R W}^{*}$ whose components are all equal to $t_{\max , R W}^{*}$. A proof of this result is provided in Appendix F.

Similarly to the results introduced in Section III-C, the set $\mathbb{T}_{\text {max,RW }}$ of all optimal solutions to (23) is a convex polytope as proved in Appendix G. Furthermore, Algorithm 2 allows the computation of an optimal solution $\mathbf{t}_{\max , \mathrm{RW}}^{m} \in \mathbb{T}_{\text {max,RW, which }}$ has components smaller than $t_{\max , \mathrm{RW}}^{*}$. In Algorithm 2 at line 1, the components of vector $\mathbf{b}_{\max }$ are defined as $b_{\max , j}=\max _{\mathbf{f} \in \mathrm{RW}} b_{j}$ (cf in Appendix G). Algorithm 2 is explained in Appendix $\mathrm{H}$ where it is pointed out that, by definition of $\mathbf{b}_{\max }$ :

$$
\mathbf{A t}_{\max } \geq \mathbf{b}, \forall \mathbf{f} \in \mathrm{RW} \quad \Longleftrightarrow \quad \mathbf{A t}_{\max } \geq \mathbf{b}_{\max }
$$

so that Algorithm 2 is mostly the same as Algorithm 1, the difference being that $\mathbf{b}_{\max }$ is used in place of $\mathbf{b}$.

From (12), since $b_{j}$ is equal to $\mathbf{c}_{j} \mathbf{f}$ minus a term independent of $\mathbf{f}$, we have:

$$
\underset{\mathbf{f} \in \mathrm{RW}}{\operatorname{argmax}} b_{j}=\underset{\mathbf{f} \in \mathrm{RW}}{\operatorname{argmax}} \mathbf{c}_{j} \mathbf{f}, \quad 1 \leq j \leq p .
$$

Eq. (26) shows that computing the maximum of $b_{j}$ over RW in (24) (i.e., computing the components of $\mathbf{b}_{\max }$ ) amounts to determining the wrench(es) $\mathbf{f}$ which maximizes the linear form $\mathbf{c}_{j} \mathbf{f}$. Note that this computation is trivial for $p+1 \leq j \leq q$ since $b_{j}$ is constant $\left(\mathbf{b}_{2}=\mathbf{t}_{\min }\right.$ in (10)). The calculation of these wrenches is discussed in Sections IV-B and IV-C in the general cases of a convex polytope RW and an ellipsoidal $\mathrm{RW}$, respectively. This calculation is then illustrated in Section IV-F in several practical examples of RW definitions. These calculations are relevant to avoid having to discretize the RW, and then to determine the smallest $\mathbf{t}_{\max }$ for all the individual wrenches $\mathbf{f}$ in this discretization. This would be highly time consuming, notably when used within a design procedure where a prescribed workspace is also discretized.

\section{B. Case of a convex polytope $R W$}

When the RW is a (bounded) convex polytope, it can be represented as the set of convex combinations of a finite number of wrenches $\mathbf{f}_{i}$ (its vertices) [47]. Consider the parallel hyperplanes $\mathbf{c}_{j} \mathbf{f}=c$, where $c$ is a scalar. Geometrically, the maximum of the linear form $\mathbf{c}_{j} \mathbf{f}$ is attained at one vertex $\mathbf{f}_{i}$ of RW, the one corresponding to the largest value of $c$ such that the hyperplane $\mathbf{c}_{j} \mathbf{f}=c$ touches the convex polytope RW. This result is well-known in linear programming (e.g., [50, Chapter 13].

Then, according to (26), the computation of $\max _{\mathbf{f} \in \mathrm{RW}} b_{j}$ in (24) amounts to calculating $b_{j}$ for all the vertices $\mathbf{f}_{i}$ of the RW and retaining the maximum value. The latter method is convenient when the vertices of RW are known and limited in number, such as illustrated in Section IV-E, as well as in Section IV-F for $\mathrm{RW}_{\text {rect }}$ and $\mathrm{RW}_{\text {square }}$. Otherwise, a linear programming problem can be solved to compute $\max _{\mathbf{f} \in \mathrm{RW}} b_{j}$.

\section{Case of an ellipsoidal $R W$}

Another common case of a convex RW is the ellipsoid. According to (26), the computation of $\max _{\mathbf{f} \in \mathrm{RW}} b_{j}$ in (24) then amounts to determining the maximum of a linear function over the ellipsoid, i.e.:

$$
\begin{array}{ll} 
& \max _{\mathbf{f} \in \mathrm{RW}} \mathbf{c}_{j} \mathbf{f} \\
\text { subject to } & (\mathbf{f}-\mathbf{e})^{T} \mathbf{E}(\mathbf{f}-\mathbf{e}) \leq 1
\end{array}
$$

where the ellipsoid is centered at $\mathbf{e}$ and $\mathbf{E}$ is a symmetric positive definite matrix. A known result (straightforward to prove with the method of Lagrange multipliers) is the following closed-form expression of the wrench $\mathbf{f}_{j}^{*}$ at which this maximum is obtained:

$$
\mathbf{f}_{j}^{*}=\mathbf{e}+\frac{\mathbf{E}^{-1} \mathbf{c}_{j}^{T}}{\sqrt{\mathbf{c}_{j} \mathbf{E}^{-1} \mathbf{c}_{j}^{T}}}
$$

and thus

$$
\max _{\mathbf{f} \in \mathrm{RW}} \mathbf{c}_{j} \mathbf{f}=\mathbf{c}_{j} \mathbf{f}_{j}^{*}=\mathbf{c}_{j} \mathbf{e}+\sqrt{\mathbf{c}_{j} \mathbf{E}^{-1} \mathbf{c}_{j}^{T}}
$$

which is useful to compute $t_{\max , \mathrm{RW}}^{*}$ in (24) since the maximum can be directly computed from $\mathbf{c}_{j}$, e and $\mathbf{E}$. This result is used in Appendix $\mathbf{J}$ to determine the wrench due to lateral disturbance forces yielding $\max _{\mathbf{f} \in \mathrm{RW}} b_{j}$. 


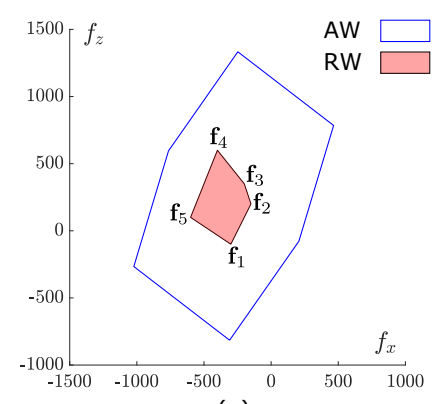

(a)

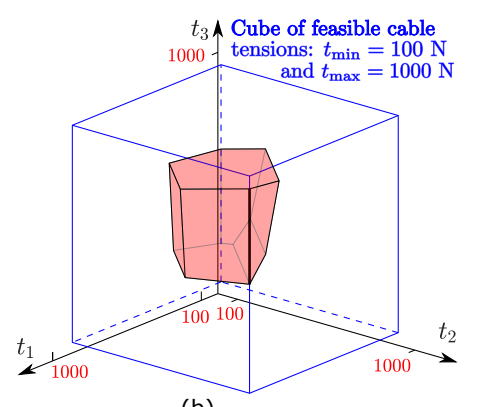

(b)
Fig. 4. (a) The AW and an example RW in the wrench space, and (b) the cube of feasible cable tensions and the image of the RW in the cable tension space. Only the subset of the image of the RW contained inside the cube of feasible cable tensions is shown.

\section{Case of a non-convex $R W$}

If the RW is not a convex set, the computation of $\max _{\mathbf{f} \in \mathrm{RW}} b_{j}$ can be done over its convex hull since:

$$
\max _{\mathbf{f} \in \mathrm{RW}} \mathbf{c}_{j} \mathbf{f}=\max _{\mathbf{f} \in \operatorname{conv}(\mathrm{RW})} \mathbf{c}_{j} \mathbf{f}
$$

where conv(RW) is the convex hull of RW defined as [47]:

$$
\operatorname{conv}(\mathrm{RW})=\left\{\mathbf{f} \mid \mathbf{f}=\sum_{k} \lambda_{k} \mathbf{f}_{k}, \mathbf{f}_{k} \in \mathrm{RW}, \lambda_{k} \geq 0, \sum_{k} \lambda_{k}=1\right\}
$$

Let $\mathbf{f}^{*}$ be the wrench in RW corresponding to the maximum of $\mathbf{c}_{j} \mathbf{f}: \mathbf{f}^{*}=\underset{\mathbf{f} \in \mathrm{RW}}{\operatorname{argmax}} \mathbf{c}_{j} \mathbf{f}$. Then, for any $\mathbf{f} \in \operatorname{conv}(\mathrm{RW})$, we have:

$$
\mathbf{c}_{j} \mathbf{f}=\sum_{k} \lambda_{k} \mathbf{c}_{j} \mathbf{f}_{k} \leq \sum_{k} \lambda_{k} \mathbf{c}_{j} \mathbf{f}^{*}=\mathbf{c}_{j} \mathbf{f}^{*}
$$

where $\mathbf{f}_{k} \in \mathrm{RW}$, the first and last equalities come from (31) and $\mathbf{c}_{j} \mathbf{f}_{k} \leq \mathbf{c}_{j} \mathbf{f}^{*}$ by definition of $\mathbf{f}^{*}$. Eq. (32) implies that $\mathbf{f}^{*}$ yields also the maximum of $\mathbf{c}_{j} \mathbf{f}$ over $\operatorname{conv}(\mathrm{RW})$, i.e.:

$$
\max _{\mathbf{f} \in \mathrm{RW}} \mathbf{c}_{j} \mathbf{f}=\mathbf{c}_{j} \mathbf{f}^{*}=\max _{\mathbf{f} \in \operatorname{conv}(\mathrm{RW})} \mathbf{c}_{j} \mathbf{f}
$$

which proves (30).

\section{E. Example and graphical interpretation}

Let us consider again the simple planar two-DOF threecable CDPR used as an example in Section III-D. With $t_{\text {min }}=100 \mathrm{~N}$ and $t_{\text {max }}=1000 \mathrm{~N}$ for each cable, and at position $P=(0.3 ; 1)$, the set of feasible cable tensions is a cube in the three-dimensional space of tensions $\mathbf{t}=\left[t_{1}, t_{2}, t_{3}\right]^{T}$ (Fig. 4 (b)). This cube is mapped by the wrench matrix $\mathbf{W}$ to the AW defined in (3). As shown in Fig. 4 (a), in this example, the wrench space is of dimensional two and the AW is thus a convex polygon, more precisely an hexagon (a twodimensional zonotope). This figure also shows a RW defined as a convex polygon whose vertices are $\mathbf{f}_{1}=[-300,-100]^{T}$, $\mathbf{f}_{2}=[-150,200]^{T}, \mathbf{f}_{3}=[-200,350]^{T}, \mathbf{f}_{4}=[-400,600]^{T}$ and $\mathbf{f}_{5}=[-600,100]^{T}(\mathrm{~N})$. The image of this RW in the tension space is shown in Fig. 4 (b). Since the nullspace of the wrench matrix $\mathbf{W}$ is not empty and, in this example, one-dimensional, this image is an unbounded convex polyhedron extending to infinity along the direction defined by a vector spanning the nullspace of W. In Fig. 4 (b), only the subset of the image of

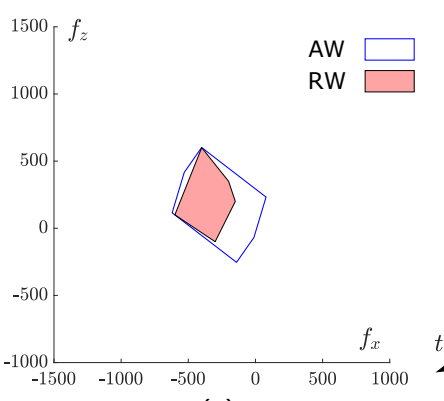

(a)

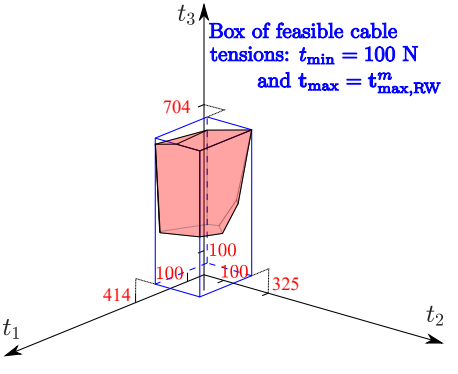

(b)
Fig. 5. The AW and the example RW in the case $\mathbf{t}_{\max }=\mathbf{t}_{\max , \mathrm{RW}}^{m}$ : (a) in the wrench space, and (b) in the cable tension space.

the RW contained inside the cube of feasible cable tensions is shown. This subset is non-empty since the RW lies inside the AW as shown in Fig. 4 (a).

Aiming to reduce the value of $t_{\max }$ in the example above, the smallest maximum tension vectors $\mathbf{t}_{\max , \mathrm{RW}}^{*}$ and $\mathbf{t}_{\max , \mathrm{RW}}^{m}$ can be calculated as explained in Section IV-A and IV-B, yielding:

$$
\mathbf{t}_{\mathrm{max}, \mathrm{RW}}^{*}=\left[\begin{array}{c}
703.87 \\
703.87 \\
703.87
\end{array}\right](\mathrm{N}) \quad \mathbf{t}_{\mathrm{max}, \mathrm{RW}}^{m}=\left[\begin{array}{l}
413.60 \\
325.46 \\
703.87
\end{array}\right](\mathrm{N}) \text {. }
$$

As illustrated in Fig 5, the smallest maximum cable tensions $\mathbf{t}_{\max }=\mathbf{t}_{\text {max }, \mathrm{RW}}^{m}$ actually yields an AW tightly enclosing the RW. Note that this AW would even more tightly enclose the RW for larger minimum tensions $t_{\min }$ (here, $t_{\min }=100 \mathrm{~N}$ has been kept constant).

\section{F. Practical RW cases}

This section introduces RW definitions related to heavy payload manipulation and which are notably of interest in the configuration design case study presented in Section V. In this case study, the CDPR is intended to work on the facade of a building in construction, operating in a quasi-static manner (for safety purposes) while carrying heavy payloads.

Two cases are considered to define the RW. In the first one, the mobile platform weight must be balanced by the cables and the vertical projection of the mobile platform center of mass (CoM) lies in a square centered at the CDPR mobile platform reference point (Section IV-F1). In the second case, in Section IV-F2, the platform is carrying heavy payloads along the building facade and the overall (platform and payload) CoM is thus shifted towards the building. In addition, disturbances are unavoidable in outdoor construction applications. The case of a RW accounting in a simple manner for lateral disturbance forces on the CDPR mobile platform is thus discussed in Section IV-F3. For each of these RWs, a closedform expression of the wrench $\mathbf{f}$ yielding the maximum of $b_{j}$ in (24) is determined. Such closed-form expressions are useful to efficiently compute (24), and thus to efficiently determine the smallest maximum cable tension vectors, since the RW does not need to be discretized.

1) First case (unloaded)-CoM projection lying in a square: Let us first consider the RW defined as the set of wrenches $\mathbf{f}=\left[f_{x}, f_{y}, f_{z}, t_{x}, t_{y}, t_{z}\right]^{T}$ allowing to balance the total mass $m$ 


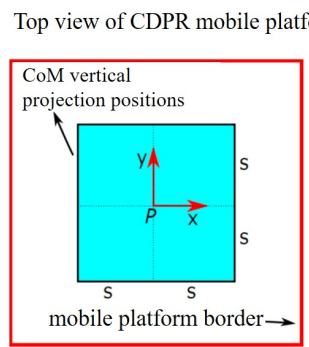

(a)

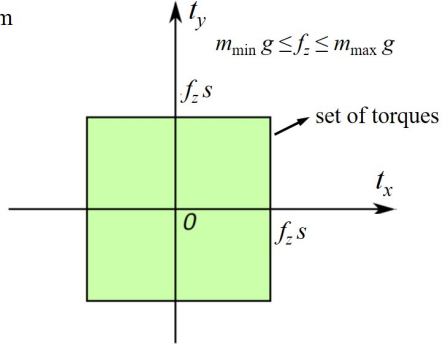

(b)
Fig. 6. (a) Set of possible positions of the CoM vertical projection (square zone), and (b) the corresponding set of torques in the wrench subspace $\left(t_{x}, t_{y}\right)$ for a given force $f_{z}$.

of the mobile platform, where $m_{\min } \leq m \leq m_{\max }$ and where the projection on the horizontal plane containing the platform reference point $P$ of the $\mathrm{CoM}$ lies in a square of dimensions $2 s \times 2 s$ centered at $P$, i.e.:

$$
\begin{aligned}
\mathrm{RW}_{\text {square }}= & \left\{\mathbf{f} \mid f_{x}=f_{y}=t_{z}=0, m_{\min } g \leq f_{z} \leq m_{\max } g,\right. \\
& \left.\left|t_{x}\right| \leq f_{z} s,\left|t_{y}\right| \leq f_{z} s\right\}
\end{aligned}
$$

where $g$ is the gravity acceleration. $f_{x}, f_{y}, f_{z}, t_{x}, t_{y}$ and $t_{z}$ are the components of $\mathbf{f}$ in a coordinate frame having a vertical axis $\mathrm{Z}$ and whose origin is the platform reference point $\mathrm{P}$.

This RW is of interest for CDPRs whose mobile platform mass and CoM position are not exactly known or varying. Figure 6 shows the square of the possible positions of the CoM vertical projection and the set of torques $t_{x}$ and $t_{y}$ required to balance the associated weight for a given value of the total mass $m, m_{\min } \leq m \leq m_{\max }$.

In order to compute (24) or $\mathbf{b}_{\max }$ in (25), $\max _{\mathbf{f} \in \mathrm{RW}} b_{j}$ must be determined for each $j$. The components of the wrench in $\mathrm{RW}_{\text {square }}$ defined in (35) yielding this maximum of $b_{j}$ (i.e., the components of the wrench defined in (26)) are given by:

$$
\begin{aligned}
& f_{x}=f_{y}=t_{z}=0 \\
& f_{z}=\left\{\begin{array}{lll}
m_{\max } g & \text { if } & c_{j f z}+s\left(\left|c_{j t x}\right|+\left|c_{j t y}\right|\right) \geq 0 \\
m_{\min } g & \text { if } & c_{j f z}+s\left(\left|c_{j t x}\right|+\left|c_{j t y}\right|\right)<0
\end{array}\right. \\
& t_{x}=\operatorname{sgn}\left(c_{j t x}\right) f_{z} s, \quad t_{y}=\operatorname{sgn}\left(c_{j t y}\right) f_{z} s
\end{aligned}
$$

where $\mathbf{c}_{j}=\left[c_{j f x}, c_{j f y}, c_{j f z}, c_{j t x}, c_{j t y}, c_{j t z}\right]$ and $\operatorname{sgn}(x)=1$ if $x \geq 0$ and $\operatorname{sgn}(x)=-1$ if $x<0$. Eq. (36) is proved in Appendix I, where the fact that $\mathrm{RW}_{\text {square }}$ is a convex polytope is used to obtain (36) from the general result stated in Section IV-B.

2) Second case (loaded)-Shifted CoM: This case is typically relevant to CDPRs carrying heavy payloads along a building facade. The total mass $m, m_{\operatorname{minl}} \leq m \leq m_{\operatorname{maxl}}$, of the platform and payload is larger than in the previous RW case $\left(m_{\operatorname{maxl}}>m_{\max }\right)$ and the CoM is shifted towards the mobile platform side to which a heavy payload is secured. A possible RW definition accounting for this shift of the overall CoM consists in choosing the platform reference point $P$ at the expected (shifted) position of the overall CoM and in considering a square projected $\mathrm{CoM}$ zone centered at $P$. However, placing the reference point at the overall CoM requires a knowledge of the detailed design of the platform and on-board components

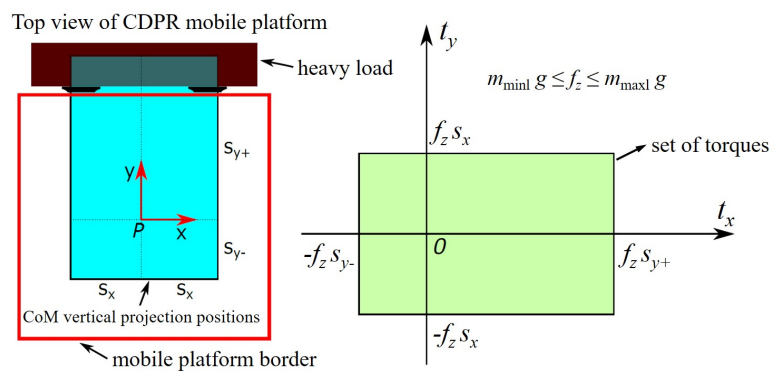

(a)

(b)

Fig. 7. (a) Set of possible positions of the CoM vertical projection (rectangular zone), and (b) the corresponding set of torques in the wrench subspace $\left(t_{x}, t_{y}\right)$ for a given force $f_{z}$.

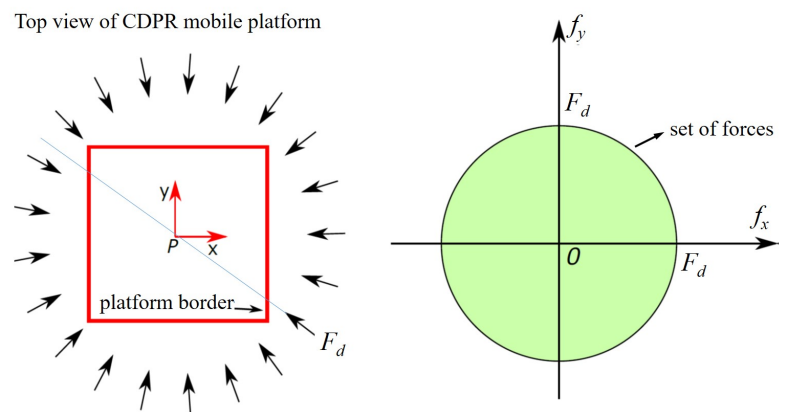

Fig. 8. (a) Directions of possible lateral disturbance forces, and (b) the corresponding set of forces in the wrench subspace $\left(f_{x}, f_{y}\right)$.

as well as of the payload mass. Alternatively, the reference point $P$ can be taken at the platform center of geometry and a shifted rectangular projected CoM zone is defined. In the case of a CDPR installed on a building facade such as illustrated in Fig. 2, the rectangular zone can be defined to be symmetric with respect to the $y$ axis and shifted with respect to the $x$ axis to account for the payload placed as shown in Fig. 7, yielding the following RW definition:

$$
\begin{aligned}
\mathrm{RW}_{\text {rect }}= & \left\{\mathbf{f} \mid f_{x}=f_{y}=t_{z}=0, m_{\text {minl }} g \leq f_{z} \leq m_{\operatorname{maxl}} g,\right. \\
& \left.-f_{z} s_{y-} \leq t_{x} \leq f_{z} s_{y+},\left|t_{y}\right| \leq f_{z} s_{x}\right\}
\end{aligned}
$$

where $s_{x}$ is the half length along $x$ of the rectangular projected CoM zone $\left(s_{x}>0\right)$, while the side lengths along the negative and positive $y$ directions are $s_{y-}$ and $s_{y+}$, respectively $\left(s_{y_{-}}>0\right.$ and $\left.s_{y+}>0\right)$.

The components of the wrench in $\mathrm{RW}_{\text {rect }}$ yielding $\max _{\mathbf{f} \in \mathrm{RW}} b_{j}$ are given by:

$$
\begin{aligned}
& f_{x}=f_{y}=t_{z}=0 \\
& f_{z}= \begin{cases}m_{\operatorname{maxl}} g & \text { if } c_{j f z}+s_{y j} c_{j t x}+s_{x}\left|c_{j t y}\right| \geq 0 \\
m_{\operatorname{minl}} g & \text { if } c_{j f z}+s_{y j} c_{j t x}+s_{x}\left|c_{j t y}\right|<0\end{cases} \\
& t_{x}=f_{z} s_{y j}, \quad t_{y}=\operatorname{sgn}\left(c_{j t y}\right) f_{z} s_{x}
\end{aligned}
$$

where $s_{y j}=\left\{\begin{aligned} s_{y+} & \text { if } c_{j t x} \geq 0 \\ -s_{y-} & \text { if } c_{j t x}<0\end{aligned}\right.$. The proof of (38) is not presented since it is similar to the one of (36) in Appendix I.

3) Disturbances and definition of two working modes:

In outdoor construction applications, disturbances such as wind or interaction forces with the robot environment are unavoidable. On the one hand, vertical disturbance forces are 
considered negligible compared to the weights of the heavy platform and payload. On the other hand, in order to account for the CDPR capabilities to balance lateral forces in a simple manner (and in quasi-static analysis), the line of action of the net lateral disturbance force $F_{d}$ is considered to pass through the reference point $P$ so that its moment at $P$ is null (Fig. 8). Accordingly, to balance these lateral disturbance forces, the following RW is defined:

$$
\mathrm{RW}_{\mathrm{distb}}=\left\{\mathbf{f} \mid \sqrt{f_{x}^{2}+f_{y}^{2}} \leq F_{d}, f_{z}=t_{x}=t_{y}=t_{z}=0\right\} .
$$

The components of the wrench in $\mathrm{RW}_{\text {distb }}$ yielding $\max _{\mathbf{f} \in \mathrm{RW}} b_{j}$ are all zero except $f_{x}$ and $f_{y}$ which are given by:

$$
f_{x}=F_{d} \frac{c_{j f x}}{\sqrt{c_{j f x}^{2}+c_{j f y}^{2}}}, \quad f_{y}=F_{d} \frac{c_{j f y}}{\sqrt{c_{j f x}^{2}+c_{j f y}^{2}}} .
$$

The proof of (40) is presented in Appendix $\mathbf{J}$ where the case of a lateral force $F_{d}$ whose line of action is not passing through $P$ is also dealt with.

Note that $\mathrm{RW}_{\text {distb }}$ is complementary to $R W_{\text {square }}$ and $\mathrm{RW}_{\text {rect }}$ since it contains non-zero lateral forces $f_{x}$ and $f_{y}$. In fact, two working modes will be considered in Section $\mathrm{V}$ in the configuration design of a CDPR intended to work on the facade of a building. In the first one, the mobile platform is unloaded, in the second one, it is loaded and, in both, lateral disturbance forces have to be balanced. Accordingly, two required wrench sets, $\mathrm{RW}_{\text {unloaded }}$ (first working mode) and $\mathrm{RW}_{\text {loaded }}$ (second working mode), are defined as follows:

$$
\begin{array}{r}
\mathrm{RW}_{\text {unloaded }}=\mathrm{RW}_{\text {square }}+\mathrm{RW}_{\text {distb }} \\
\mathrm{RW}_{\text {loaded }}=\mathrm{RW}_{\text {rect }}+\mathrm{RW}_{\text {distb }}
\end{array}
$$

where + denotes the Minkowski sum of two sets. According to (36), (38) and (40), the components of the wrench in $\mathrm{RW}_{\text {unloaded }}$ yielding $\max _{\mathbf{f} \in \mathrm{RW}} b_{j}$ are:

$$
\begin{aligned}
& f_{x}=F_{d} \frac{c_{j f x}}{\sqrt{c_{j f x}^{2}+c_{j f y}^{2}}}, f_{y}=F_{d} \frac{c_{j f y}}{\sqrt{c_{j f x}^{2}+c_{j f y}^{2}}}, \\
& f_{z}=\left\{\begin{array}{lll}
m_{\max } g & \text { if } & c_{j f z}+s\left(\left|c_{j t x}\right|+\left|c_{j t y}\right|\right) \geq 0 \\
m_{\min } g & \text { if } & c_{j f z}+s\left(\left|c_{j t x}\right|+\left|c_{j t y}\right|\right)<0
\end{array}\right. \\
& t_{x}=\operatorname{sgn}\left(c_{j t x}\right) f_{z} s, \quad t_{y}=\operatorname{sgn}\left(c_{j t y}\right) f_{z} s, \quad t_{z}=0
\end{aligned}
$$

and those in $\mathrm{RW}_{\text {loaded }}$ yielding $\max _{\mathbf{f} \in \mathrm{RW}} b_{j}$ are:

$$
\begin{aligned}
& f_{x}=F_{d} \frac{c_{j f x}}{\sqrt{c_{j f x}^{2}+c_{j f y}^{2}}}, f_{y}=F_{d} \frac{c_{j f y}}{\sqrt{c_{j f x}^{2}+c_{j f y}^{2}}}, \\
& f_{z}= \begin{cases}m_{\max 1} g & \text { if } c_{j f z}+s_{y j} c_{j t x}+s_{x}\left|c_{j t y}\right| \geq 0 \\
m_{\operatorname{minl}} g & \text { if } c_{j f z}+s_{y j} c_{j t x}+s_{x}\left|c_{j t y}\right|<0\end{cases} \\
& t_{x}=f_{z} s_{y j}, \quad t_{y}=\operatorname{sgn}\left(c_{j t y}\right) f_{z} s_{x}, \quad t_{z}=0 .
\end{aligned}
$$

\section{G. Summary and performance index definition}

The smallest maximum cable tension satisfying the wrenchfeasibility constraints in (2) is a relevant performance index to be minimized in a CDPR configuration design optimization. In the case study of Section $\mathrm{V}$, among the optimal solutions $\mathbf{t}_{\max , \mathrm{RW}}^{*}$ and $\mathbf{t}_{\max , \mathrm{RW}}^{m}$ to (23), the vector $\mathbf{t}_{\max , \mathrm{RW}}^{*}$ whose components are all equal to $t_{\max , \mathrm{RW}}^{*}$ given in (24) is selected since the maximum cable tension is desired to be the same for all the cables of the designed CDPR.

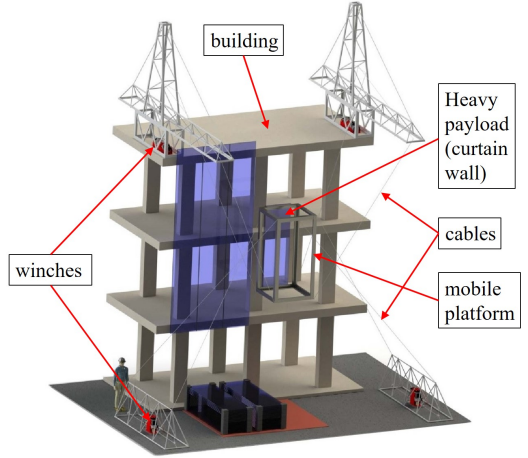

Fig. 9. CDPR working over a building facade to install curtain walls.

The expression of the smallest maximum cable tension $t_{\text {max }, \mathrm{RW}}^{*}$ in (24) has been obtained for a given pose of the CDPR mobile platform (for a given wrench matrix $\mathbf{W}$ ). In CDPR configuration optimization, $t_{\max , \mathrm{RW}}^{*}$ must usually be calculated over a prescribed workspace $\mathbb{W}$. In particular, the performance index in Section $\mathrm{V}$ is chosen to be the smallest maximum cable tension $t_{\max }^{*} \mathbb{W}$ such that wrench-feasibility constraints are satisfied across $\mathbb{W}$, i.e.:

$$
t_{\max , \mathbb{W}}^{*}=\max _{\mathbb{W}} t_{\max , \mathrm{RW}}^{*}=\max _{\mathbb{W}} \max _{j \in J_{+}}\left(\frac{\max _{\mathbf{f} \in \mathrm{RW}} b_{j}}{\sum_{i=1}^{m} a_{j i}}\right) .
$$

Note that in (44), considering the two working modes defined in IV-F 3) $\left(\mathrm{RW}=\mathrm{RW}_{\text {unloaded }}\right.$ or $\left.\mathrm{RW}=\mathrm{RW}_{\text {loaded }}\right)$, either (42) or (43) are used to computed $\max _{\mathbf{f} \in \mathrm{RW}} b_{j}$. If both working modes shall be taken into account, which is the case in Section $\mathrm{V}, t_{\max }^{*} \mathbb{W}$ is calculated for each working mode separately and the maximum of these two values is retained since it is the smallest maximum tension allowing the satisfaction of the wrench-feasibility constraints of the two working modes.

\section{DESIGN OF A CDPR CONFIGURATION}

In this case study, the CDPR mobile platform is expected to manipulate heavy loads, e.g., curtain walls, across the facade of a building in construction, such as illustrated in Fig. 9. The CDPR motions are quasi-static (relatively slow) and the loads may be off-centered. Motions along the building facade vertical plane and perpendicular to it are both necessary. Rotations of the platform, possibly of small amplitudes, must also be controlled in order to adjust the module pose during its installation and for picking it from a storage place on the ground. Accordingly, the positioning accuracy of the CDPR and its capability to balance shifted loads are important. These requirements justify the consideration of fully-constrained 6DOF CDPRs $(n=6)$ since wrench capabilities and positioning accuracy along all six DOFs are needed.

This section presents the design of the configuration of such a 6-DOF CDPR where configuration refers to the positions of the cable attachment points on the mobile platform, the positions of the cable drawing points on the fixed base and the cable arrangement between these two sets of points. The CDPR configuration must be carefully designed in order to 


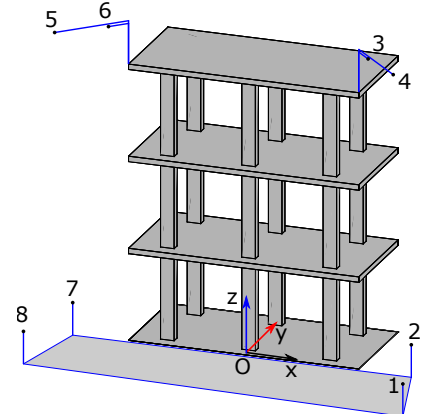

(a)

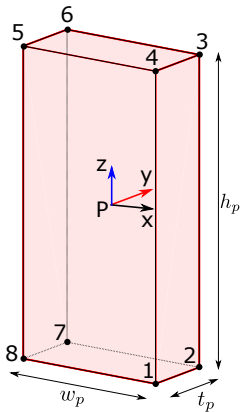

(b)
Fig. 10. (a) Fixed base geometry, and (b) mobile platform geometry.

obtain a suitable wrench-feasible workspace. Eight cables $(m=8)$ are considered in this work since this number of cables allows cable arrangements with symmetry properties which lead generally to more homogeneous performances across the workspace [6], [7], [9], [10].

A configuration design methodology similar to the one introduced in [6] is used. It consists of two phases which can be summarized as follows:

- Phase 1: Generation and test of a large number of possible CDPR configurations in order to define a set of candidate configurations. The tests consist in verifying that each generated CDPR configuration satisfies a number of constraints. The feasible configurations are sorted according to their smallest maximum cable tension $t_{\max , \mathbb{W}}^{*}$ defined in (44) in Section IV-G.

- Phase 2: Among the feasible CDPR configurations found in Phase 1 , the ones having the smallest values of $t_{\max }^{*}, \mathbb{W}$ are retained. The number of retained configurations is defined by the user. These configurations serve as initial guesses for local constrained optimization problems and each of them is thereby refined. The objective function of the optimization is the performance index defined in (44).

The differences with the procedure in [6] lie in the constraint and performance index definitions and in the fact that several CDPR configurations are optimized in Phase 2. Similarly to [6], the main limitations of this procedure are that only a finite set of poses in the CDPR prescribed workspace are considered and the overall computation time increases proportionally to the number $N_{w}$ of poses in this set and to the number $N_{c}$ of tested configurations, i.e., $O\left(N_{w} N_{c}\right)$ using the big $O$ notation. These limitations may be mitigated by using a relatively small number of poses and by verifying, at the end of the design procedure, that the best configurations found satisfy the constraints across the prescribed workspace, e.g., by means of a fine discretization of the latter (cf. in Section V-C).

\section{A. Phase 1: Candidate CDPR configurations}

1) Base and platform geometries: Inputs to the configuration design methodology include the fixed base and mobile platform geometries shown in Fig. 10. The corresponding cable drawing points at the base and attachment points on the platform are given in Table I and III, respectively. The
TABLE I

DRAWING POINTS COORDINATES

\begin{tabular}{|c|c|c|c|c|}
\cline { 2 - 5 } \multicolumn{1}{c|}{} & \multicolumn{4}{c|}{ Drawing points } \\
\cline { 2 - 5 } \multicolumn{1}{c|}{} & 1 & 2 & 3 & 4 \\
\hline$x$ & $w / 2+p_{1 x}$ & $w / 2+p_{2 x}$ & $w / 2+p_{3 y} \tan \left(p_{34 \alpha}\right)$ & $w / 2+p_{4 y} \tan \left(p_{34 \alpha}\right)$ \\
\hline$y$ & $-p_{1 y}$ & $-p_{2 y}$ & $-p_{3 y}$ & $-p_{4 y}$ \\
\hline$z$ & $p_{12 z}$ & $p_{12 z}$ & $h+p_{3 z}$ & $h+p_{3 z}+p_{4 z}$ \\
\hline
\end{tabular}

TABLE II

BOUNDS ON DRAWING POINT DIMENSIONAL PARAMETERS

\begin{tabular}{|c|c|c|c|c|c|c|c|c|}
\cline { 2 - 9 } \multicolumn{1}{c|}{} & $p_{1 x}$ & $p_{1 y}$ & $p_{2 x}$ & $p_{2 y}$ & $p_{3 y}$ & $p_{3 z}$ & $p_{4 y}$ & $p_{34 \alpha}$ \\
\hline units & \multicolumn{9}{|c|}{$(m)$} & $\left(^{\circ}\right)$ \\
\hline min. & 0 & 3 & 0 & 0 & 0.3 & 1 & 2 & 0 \\
\hline max. & 2 & 5 & 2 & 2 & 1 & 1.5 & 3 & 45 \\
\hline
\end{tabular}

bounds on the corresponding dimensional parameters are given in Table II and IV.

The building facade has a height $h$ of $10.4 \mathrm{~m}$ and a width $w$ of $8.5 \mathrm{~m}$. The cable drawing points are defined in such a way that the CDPR workspace can cover the building facade while limiting their extension outside of the building. The drawing point positions are symmetric with respect to the vertical $O-y z$ plane at the middle of the building. Hence, Table I only defines the coordinates of the first four drawing points since the coordinates of the four other ones can be deduced by symmetry. Some dimensional parameters have constant values. The height of the drawing points close to the ground is fixed at $p_{12 z}=1.2 \mathrm{~m}$ and the drawing point 4 is slightly higher than point $3\left(p_{4 z}=0.1 \mathrm{~m}\right)$ to facilitate cable routing.

As shown in Fig. 10, the CDPR mobile platform geometry is a box of dimensions $w_{p} \times t_{p} \times h_{p}$. Only the first four attachment points are defined since the other four ones are obtained by symmetry with respect to the $P-y z$ plane. The notations $p_{i}^{+}$and $p_{i}^{-}$used in Table III are defined as:

$$
p_{i}^{+}=\left\{\begin{array}{l}
p_{i} \text { if } p_{i}>0 \\
0 \text { if } p_{i} \leq 0
\end{array}, \quad p_{i}^{-}=\left\{\begin{array}{l}
0 \text { if } p_{i} \geq 0 \\
p_{i} \text { if } p_{i}<0
\end{array} .\right.\right.
$$

To accommodate cables up to $15 \mathrm{~mm}$ in diameter, routing pulleys of diameter $30 \mathrm{~cm}$ are considered [51]. The output pulley kinematics is then non negligible and the method presented in [52] is used to account for the output pulley kinematics in computing the wrench matrix $\mathbf{W}$.

2) Generation of candidate CDPR configurations: The cable arrangements between the drawing and attachment points are generated as follows. Each drawing point is linked to one attachment point not already linked to another drawing point. The symmetries of the base and platform with respect to their $y z$ planes are reflected into the cable arrangements, i.e., if the drawing point $i$ is linked to the attachment point $j$, the symmetric of point $i$ is linked to the symmetric of point $j$. Besides, cable arrangements that clearly lead to collisions between some cables and the platform are discarded. Thereby, 24 different cable arrangements are generated. In addition, each base and platform dimensional parameter can take its minimum and maximum values (as defined in Tables II and IV), as well a few values in between. Thereby, a total of 746496 candidate CDPR configurations were generated in the present case study. 
TABLE III

ATTACHMENT POINTS COORDINATES IN THE MOBILE PLATFORM FRAME

\begin{tabular}{|c|c|c|c|c|}
\cline { 2 - 5 } \multicolumn{1}{c|}{} & \multicolumn{4}{c|}{ Attachment points } \\
\cline { 2 - 5 } \multicolumn{1}{c|}{} & 1 & 2 & 3 & 4 \\
\hline$x=w_{p} / 2 \times$ & 1 & 1 & 1 & 1 \\
\hline$y=t_{p} / 2 \times$ & $-1+p_{1}^{+}$ & $-1-p_{2}^{+}$ & $1-p_{3}^{+}$ & $-1+p_{4}^{+}$ \\
\hline$z=h_{p} / 2 \times$ & $-1-p_{1}^{-}$ & $-1-p_{2}^{-}$ & $1+p_{3}^{-}$ & $-1+p_{4}^{-}$ \\
\hline
\end{tabular}

TABLE IV

BOUNDS ON ATTACHMENT POINT DIMENSIONAL PARAMETERS

\begin{tabular}{|c|c|c|c|c|c|c|c|}
\cline { 2 - 8 } \multicolumn{1}{c|}{} & $w_{p}$ & $t_{p}$ & $h_{p}$ & $p_{1}$ & $p_{2}$ & $p_{3}$ & $p_{4}$ \\
\hline units & \multicolumn{3}{c}{$(m)$} & & \multicolumn{4}{c|}{} \\
\hline min. & 1.5 & 1 & 2 & -1 & -1 & -1 & -1 \\
\hline max. & 1.5 & 1.5 & 3 & 1 & 1 & 1 & 1 \\
\hline
\end{tabular}

3) Prescribed workspace: The CDPR mobile platform is required to span the surface in front of the building within a limited depth. Hence, the prescribed workspace $\mathbb{W}$ is located in front of the building, spanning the whole facade while being relatively flat. In both phases of the configuration design methodology, the objective function defined in (44) and the different constraints (defined below) are evaluated at a set of discrete poses inside this workspace.

This set of poses is defined as follows. Taking advantage of the building and CDPR symmetries with respect to the middle $y z$ plane, eighteen workspace points are considered, these points being distributed on only one side of the building as shown in Fig. 11. Along the $x$ axis, the points are distributed between the middle and side of the building. Along the $y$ axis, the points are distributed on two parallel planes, one at distance of $0.4+t_{p} / 2 \mathrm{~m}$ from the building facade ("installation distance") and the other one at $1+t_{p} / 2 \mathrm{~m}$ (load "transportation distance"). Along the $z$ axis, the lower points are at $1.2 \mathrm{~m}$ above the ground and correspond to the mobile platform being positioned in front of the building ground floor, i.e., at the bottom of $\mathbb{W}$. The upper points are at $1.2 \mathrm{~m}$ below the top which corresponds to the last floor, i.e., to the top of $\mathbb{W}$. Moreover, at each of these points, platform orientation angles about the $z$ axis of $-2^{\circ}, 0^{\circ}$ and $2^{\circ}$ are considered to accommodate possible building construction deviations along the facade. On overall, 54 poses inside $\mathbb{W}$ are thus used to test the constraints and calculate the objective function. This discrete set comprises poses located inside and on the boundary of $\mathbb{W}$. While the computation time of the various steps of the configuration design methodology is (approximately) proportional to the number of poses in this discretization of $\mathbb{W}$, in our experience, it was not necessary to consider a discrete set containing a large number of poses as long as poses on the boundary of the prescribed workspace were included. Note also that the final configuration obtained by the design procedure can be tested with a much finer discretization in order to check the feasibility of the different constraints and compute the values of the objective function across the prescribed workspace (Section V-C).

4) Definition of the constraints: The CDPR mobile platform can be subjected to external disturbances and the CDPR has a relatively low stiffness along the direction perpendicular to the building facade ( $y$ axis). Hence, the first constraint is

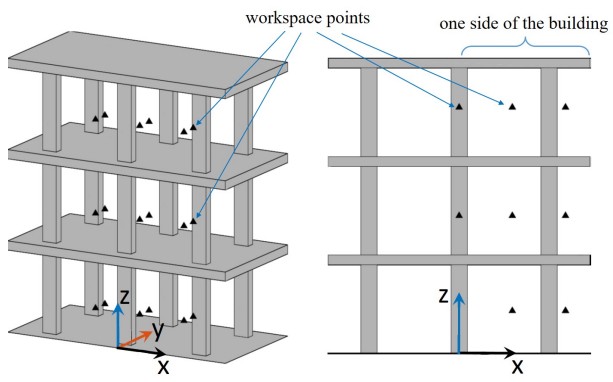

Fig. 11. The workspace points considered in the CDPR configuration design procedure.

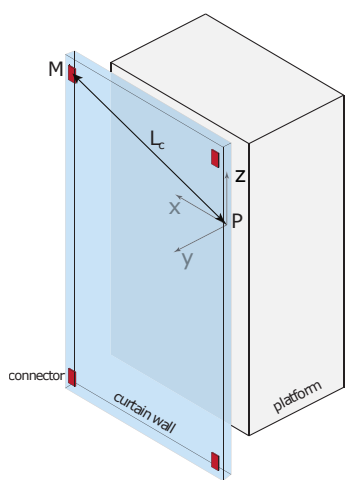

Fig. 12. Definition of $L_{c}$ in the example of curtain wall module positioning. The curtain wall needs to be fixed to the building facade by means of brackets and connectors. Point $M$ defines the position of one of the connectors.

the displacement of the mobile platform due to variations of the external wrench which must remain smaller than $10 \mathrm{~mm}$. A point $M$ of the platform, whose positioning accuracy is considered critical, is chosen in order to define this displacement, denoted $\delta M$. For instance, if the CDPR displaces and positions curtain wall modules, the point $M$ is defined as the position of one of the module connectors such as illustrated in Fig. 12. For the disturbances, a maximal wind speed of $15 \mathrm{~m} / \mathrm{s}$ applied over the surface of a curtain wall module is considered (with a simple model for the calculation of the resulting lateral force) and the worst case payload variation is considered to be a modification of $30 \mathrm{~kg}$ located at $400 \mathrm{~mm}$ away from the platform reference point $P$. These disturbances yield a variation of the external wrench, which is used to compute the displacement $\delta M$ by means of the CDPR stiffness matrix. The latter is homogenized with the procedure presented in [53] using the characteristic length $L_{c}=\overline{P M}$ shown in Fig. 12. The cable stiffness value is EA $=8.5 \mathrm{e} 6 \mathrm{~N}$ obtained by stress-strain measurements on a sample of a steel cable of diameter $12 \mathrm{~mm}$.

The second constraint is the absence of cable collisions which includes here cable-cable and cable-environment collisions. The cable-cable collision is tested by computing the distance between two cables considering cable sagging as negligible and for a maximum cable diameter of $20 \mathrm{~mm}$. The cable-environment collision test consists in checking the collision between the cables and the platform and between the cables and a curtain wall module fixed on one side of the mobile platform as illustrated in Fig. 12. 

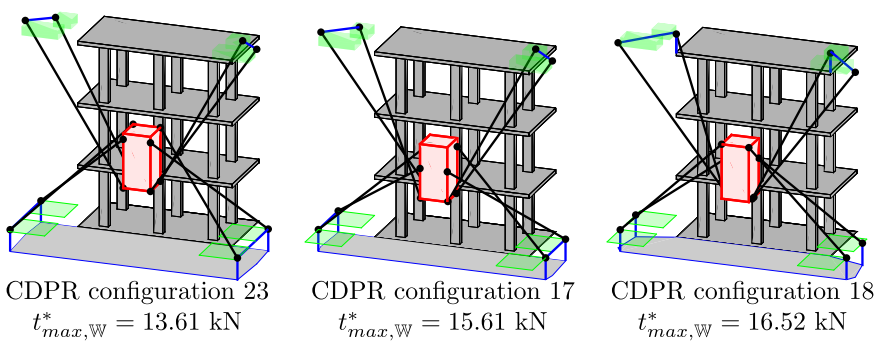

Fig. 13. CDPR configurations obtained after the optimization made in Phase 2 of the configuration design methodology. Each one has a different cable arrangement. Configuration 23 is the best one since it possesses the smallest value of $t_{\max , \mathbb{W}}^{*}$

5) Evaluation of the candidate CDPR configurations: The configurations generated in step 2) which have no cable collision and a platform displacement $\delta M<10 \mathrm{~mm}$ for all poses in the discretized workspace $\mathbb{W}$, are classified according to the value of their smallest maximum cable tension $t_{\text {max. }}^{*}$ defined in (44). The smaller is $t_{\text {max, }}^{*}$, the better is the configuration. In these calculations, the following values have been used: $m_{\min }=150 \mathrm{~kg}, m_{\max }=500 \mathrm{~kg}, m_{\operatorname{minl}}=350 \mathrm{~kg}$, and $m_{\operatorname{maxl}}=1000 \mathrm{~kg}$. Considering different possible orientations of the mobile platform, the dimensions of the sets of possible CoM positions have been set as $s=300 \mathrm{~mm}, s_{x}=300 \mathrm{~mm}$, $s_{y-}=300 \mathrm{~mm}$, and $s_{y+}=t_{p} / 2$. Furthermore, the net lateral disturbance force is $F_{d}=500 \mathrm{~N}$ and the minimum cable tension is $t_{\min }=200 \mathrm{~N}$.

Among the 746496 candidate configurations generated in step 2), 730 configurations are found to be feasible with respect to the constraints. These configurations are sorted in ascending order of their values of $t_{\max , \mathbb{W}}^{*}$. It is noticed that, out of 24 different cable arrangements obtained in step 2), the remaining 730 configurations comprise only four different cable arrangements.

\section{B. Phase 2: Refinement by optimization}

Each feasible configuration resulting from Phase 1 can be used as an initial guess of an optimization problem and is thereby refined. The optimization problem is non-linear and can have numerous local minima, which is the rationale of considering multiple initial guesses. The problem is formulated as follows:

$$
\begin{aligned}
\min _{\mathbf{x}_{d}, \mathbf{x}_{a}} & t_{\text {max, } \mathbb{W}}^{*} \\
\text { subject to } & \left\{\begin{array}{l}
\text { absence of cable collisions over } \mathbb{W} \\
\text { displacement constraint over } \mathbb{W}
\end{array}\right. \\
& \mathbf{x}_{d}^{m} \leq \mathbf{x}_{d} \leq \mathbf{x}_{d}^{M}, \quad \mathbf{x}_{a}^{m} \leq \mathbf{x}_{a} \leq \mathbf{x}_{a}^{M}
\end{aligned}
$$

where the vectors $\mathbf{x}_{d}$ and $\mathbf{x}_{a}$ contain the dimensional parameters defining the cable drawing points and attachment points, respectively. These parameters are defined in Table II and IV. The vectors $\mathbf{x}_{d}^{m}, \mathbf{x}_{d}^{M}, \mathbf{x}_{a}^{m}$ and $\mathbf{x}_{a}^{M}$ contain the dimensional parameter lower and upper bounds which are also defined in these tables. In (46), $t_{\mathrm{max}, \mathbb{W}}^{*}$ is defined in (44) and thereby includes wrench-feasibility constraints (RWs) defined with mass, dimension and force values equal to those in Section V-A 5). The wording "over $\mathbb{W}$ " in the optimization problem (46)

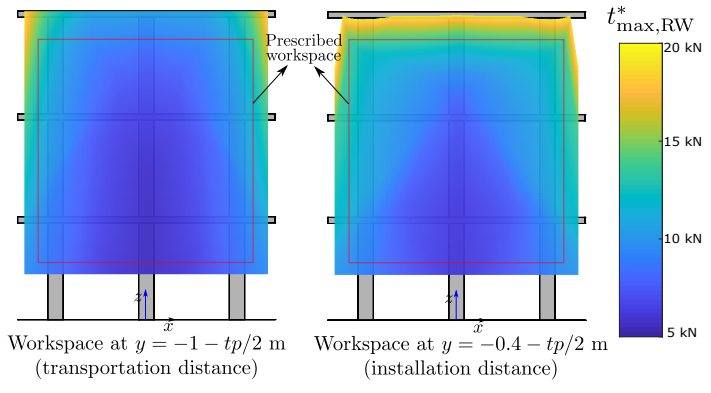

Fig. 14. The smallest maximum cable tension $t_{\max , \mathrm{RW}}^{*}$ of configuration 23 evaluated in two vertical cross sections of an extension of the prescribed workspace $\mathbb{W}$.

means for the 54 poses of the discretized workspace $\mathbb{W}$ defined in Section V-A 3). The displacement constraint in (46) is $\delta M<10 \mathrm{~mm}$ as detailed in Section V-A 4). The results of Phase 2 are discussed in the next section.

\section{Results}

The optimization in (46) can be applied with the 730 configurations retained in Phase 1 as initial guesses. However, as confirmed by experience, compared to the best CDPR configurations found in Phase 1, using as an initial guess a CDPR configuration which is low in the ranking often leads to local minimum with a larger value of $t_{\max . \mathbb{W}}^{*}$. Hence, in the results presented here, ten configurations for each of the cable arrangements remaining after Phase 1 were optimized using (46), which require several hours of computation time on a standard computer (Intel Core i7- 7820HQ @ 2.9 GHZ 16 GB RAM).

Besides, out of the four different cable arrangements remaining after Phase 1, one has values of $t_{\mathrm{max}, \mathbb{W}}^{*}$ which turned out to be sensitive to uncertainties on the dimensional parameters. Such a significant degradation of CDPR performance due to uncertainties has previously been noticed in [54]. Inspired from this previous work, the method to test sensitivity to uncertainties used in the present paper is presented in Appendix K. The configurations having this cable arrangement were thus discarded. For each of the remaining three cable arrangements, the best CDPR configuration, i.e., the one with smallest value of $t_{\max , \mathbb{W}}^{*}$, is shown in Fig. 13. The green areas in this figure are the zones where the drawing points can be located (according to Tables I and II).

Among the three configurations shown in Fig. 13, the best one is configuration 23 with $t_{\max , \mathbb{W}}^{*}=13.61 \mathrm{kN}$. The largest values of the components of $\mathbf{t}_{\text {max.RW }}^{m}$ for configuration 23, computed with Algorithm 2 of SectionIV-A across the prescribed workspace $\mathbb{W}$, are:

$$
\max _{\mathbb{W}}\left(\mathbf{t}_{\max , \mathrm{RW}}^{m}\right)=\left[\begin{array}{c}
13560 \\
13560 \\
7940 \\
13610 \\
13610 \\
7940 \\
13560 \\
13560
\end{array}\right]
$$


where the max is taken component by component. These values show that for the optimal configuration 23, the maximum cable tensions in two cables (number 3 and 6) can be significantly smaller than those in the other cables. The other smallest maximum cable tensions are equal or similar across $\mathbb{W}$ because of symmetries in the definition of this CDPR configuration. Since only 54 poses of the discretized workspace $\mathbb{W}$ were considered in phases 1 and 2, configuration 23 was verified to satisfy the constraints-absence of cable collisions and maximum displacement constraint $\delta M<10 \mathrm{~mm}$-across the prescribed workspace $\mathbb{W}$ by means of a fine discretization of $\mathbb{W}$. Moreover, the values of $t_{\mathrm{max}, \mathrm{RW}}^{*}$ (defined in (24)) for all poses of a fine discretization of an extension of $\mathbb{W}$ have also been computed. The results of these computations are shown in Fig. 14 for two vertical cross sections of the extended workspace. It can be seen that the optimal value $t_{\max , \mathbb{W}}^{*}=13.61 \mathrm{kN}$ obtained in phase 2 is valid everywhere in the prescribed workspace $\mathbb{W}$. Otherwise, or if the constraints were not satisfied across $\mathbb{W}$, a discretization of $\mathbb{W}$ containing a larger number of poses should be considered in Phases 1 and 2 of the configuration design methodology.

It can be noted that configuration 23 is different from the best one obtained in our preliminary works presented in [42] which can be explained by different definitions of the RWs, of the dimensional parameters and of the constraints. The difference in the best configuration obtained also illustrates that the CDPR performances are sensitive to the choice of its configuration.

In the framework of the project Hephaestus [1], based on configuration 23 shown in Fig. 13, the CDPR prototype shown in Fig. 1 has been designed and built. The accompanying video shows the first tests of this prototype. The latter is intended to manipulate curtain wall modules along a building facade and to position a manipulator placed on-board its mobile platform to perform various tasks related to the installation of the curtain walls. These experimental demonstrations validate the usefulness and performance of the CDPR configuration found by minimizing the smallest maximum cable tension. It can be noted that the actual masses, $973 \mathrm{~kg}$ and $1323 \mathrm{~kg}$ without and with the curtain wall module secured to the platform, respectively, are significantly larger than initially expected $\left(m_{\max }=500 \mathrm{~kg}\right.$ and $m_{\operatorname{maxl}}=1000 \mathrm{~kg}$ in Section V-A).

\section{CONCLUSION}

This paper contributed to the analysis and design of cabledriven parallel robots by disclosing means of computing smallest maximum cable tension vectors allowing, by construction, wrench-feasibility constraints to be satisfied. The problem of determining such maximum tension vectors was formulated as a minimization problem with linear inequality constraints. The set of optimal solutions to this problem was shown to be a convex polytope and, thus, it contains infinitely many solutions. Hence, there exists various smallest maximum tension vectors allowing a required wrench set to be generated with feasible cable tensions and the calculations of two different smallest maximum tension vectors were presented. The first one has all its components equal to the smallest maximum tension value $t_{\max }^{*}$. The second one has several components smaller than this value since it is calculated as the solution vector having the least possible value for each of its components. An explicit expression of $t_{\max }^{*}$ in terms of the linear inequality constraints of the proposed minimization problem was also introduced. Moreover, the general case of a wrench set, including wrench sets defined as polytopes and ellipsoids, was addressed. For several practical wrench set definitions, closedform expressions of the components of wrenches permitting an efficient computation of the smallest maximum tension vectors were also provided. These contributions were applied to the configuration optimization of a CDPR installed on the facade of a building to manipulate heavy payloads. Based on the resulting best configuration, a 6-DOF large-dimension CDPR was built and used for testing and demonstration purposes.

In the configuration optimization case study presented in this paper, only the smallest maximum tension vector which has all its components equal was used. The other smallest maximum tension vector $\mathbf{t}_{\max , \mathrm{RW}}^{m}$, whose components are not all equal, may notably prove useful for non-symmetric CDPR configurations or non-symmetric required wrench set formulations. In such cases, the computation of the components of $\mathbf{t}_{\text {max,RW }}^{m}$ across the workspace may lead to significant differences in the individual smallest maximum cable tensions. In an extension of the present work, smallest maximum force or torque vectors having components not all equal should also be relevant in the case of parallel robots with hybrid actuation where all actuators are not of the same type, or of the same size.

\section{ACKNOWLEDGMENT}

This work was supported by the European Union's H2020 Program (H2020/2014-2020) under the grant agreement No. 732513 (Hephaestus project).

\section{REFERENCES}

[1] [Online]. Available: www.hephaestus-project.eu

[2] A. Pott, Cable-Driven Parallel Robots, Theory and Application. Springer, 2018.

[3] R. R. Thompson and M. S. Blackstone, "Three-dimensional moving camera assembly with an informational cover housing," 2005, US Patent $6,873,355$

[4] J. Albus, R. Bostelman, and N. Dagalakis, "The NIST robocrane," Journal of Field Robotics, vol. 10, no. 5, pp. 709-724, 1993.

[5] R. Nan, "Five hundred meter aperture spherical radio telescope (FAST)," Science in China series G, vol. 49, no. 2, pp. 129-148, 2006.

[6] M. Gouttefarde, J.-F. Collard, N. Riehl, and C. Baradat, "Geometry selection of a redundantly actuated cable-suspended parallel robot," IEEE Transactions on Robotics, vol. 31, no. 2, pp. 501-510, 2015.

[7] A. Pott, H. Mütherich, W. Kraus, V. Schmidt, P. Miermeister, and A. Verl, "IPAnema: a family of cable-driven parallel robots for industrial applications," in Cable-Driven Parallel Robots. Springer, 2013, pp. 119-134.

[8] T. Rasheed, P. Long, D. Marquez-Gamez, and S. Caro, "Available Wrench Set for Planar Mobile Cable-Driven Parallel Robots," in 2018 IEEE International Conference on Robotics and Automation (ICRA). IEEE, may 2018, pp. 962-967.

[9] T. Bruckmann, W. Lalo, K. Nguyen, and B. Salah, "Development of a storage retrieval machine for high racks using a wire robot," in Proc. ASME International Design Engineering Technical Conferences, no. DETC2012-70389, Chicago, IL, USA, 2012.

[10] P. Miermeister et al, "The CableRobot simulator: Large scale motion platform based on cable robot technology," in Proc. IEEE/RSJ Int. Conf. Intelligent Robots and Systems (IROS), Daejeon, Korea, 2016, pp. 30243029 . 
[11] E. Barnett and C. Gosselin, "Large-scale 3D printing with a cablesuspended robot," Additive Manufacturing, vol. 7, pp. 27-44, 2015.

[12] P. Bosscher, A. T. Riechel, and I. Ebert-Uphoff, "Wrench-feasible workspace generation for cable-driven robots," IEEE Transactions on Robotics, vol. 22, no. 5, pp. 890-902, 2006.

[13] M. Gouttefarde, D. Daney, and J.-P. Merlet, "Interval-analysis-based determination of the wrench-feasible workspace of parallel cable-driven robots," IEEE Transactions on Robotics, vol. 27, no. 1, pp. 1-13, 2011.

[14] S. Kawamura, H. Kino, and C. Won, "High-speed manipulation by using parallel wire-driven robots," Robotica, vol. 18, pp. 13-21, 2000.

[15] A. Fortin-Côté et al, "On the design of a novel cable-driven parallel robot capable of large rotation about one axis," in Cable-Driven Parallel Robots, C. Gosselin, P. Cardou, T. Bruckmann, and A. Pott, Eds. Springer, 2017.

[16] E. Stump and V. Kumar, "Workspaces of cable-actuated parallel manipulators," ASME J. Mech. Des., vol. 128, no. 1, pp. 159-167, 2006.

[17] M. Gouttefarde and C. M. Gosselin, "Analysis of the wrench-closure workspace of planar parallel cable-driven mechanisms," IEEE Transactions on Robotics, vol. 22, no. 3, pp. 434-445, Jun. 2006.

[18] G. Abbasnejad, J. Eden, and D. Lau, "Generalized ray-based lattice generation and graph representation of wrench-closure workspace for arbitrary cable-driven robots," IEEE Transactions on Robotics, vol. 35 , no. 1, pp. 147-161, 2019.

[19] S. Tadokoro, S. Nishioka, T. Kimura, M. Hattori, T. Takamori, and K. Maeda, "On fundamental design of wire configurations of wire-driven parallel manipulators with redundancy," in ASME Japan/USA symposium on flexible automotion, vol. 1, 1996, pp. 151-158.

[20] S. Perreault and C. M. Gosselin, "Cable-driven parallel mechanisms: Application to a locomotion interface," ASME J. Mech. Des., vol. 130, no. 10 , Oct. 2008.

[21] K. Azizian and P. Cardou, "The dimensional synthesis of planar parallel cable-driven mechanisms through convex relaxations," ASME Journal of Mechanisms and Robotics, vol. 4, no. 3, Aug. 2012

[22] _ _ "The dimensional synthesis of spatial cable-driven parallel mechanisms," ASME Journal of Mechanisms and Robotics, vol. 5, no. 4, Nov. 2013.

[23] G. Abbasnejad, J. Yoon, and H. Lee, "Optimum kinematic design of a planar cable-driven parallel robot with wrench-closure gait trajectory," Mechanism and Machine Theory, vol. 99, pp. 1-18, 2016.

[24] R. Kurtz and V. Hayward, "Dexterity measures with unilateral actuation constraints: the $\mathrm{n}+1$ case," Advanced robotics, vol. 9, no. 5, pp. 561577, 1994.

[25] J. Eden, D. Lau, Y. Tan, and D. Oetomo, "Unilateral manipulability quality indices: Generalized manipulability measures for unilaterally actuated robots," ASME Journal of Mechanical Design, vol. 141, pp. $1-11,2019$.

[26] C. B. Pham, S. H. Yeo, G. Yang, and I.-M. Chen, "Workspace analysis of fully restrained cable-driven manipulators," Robotics and Autonomous Systems, vol. 57, no. 9, pp. 901-912, 2009.

[27] X. Tang and R. Yao, "Dimensional design of the six-cable driven parallel manipulator of FAST," ASME J. Mech. Des., vol. 133, no. 11, Nov. 2011.

[28] M. Anson, A. Alamdari, and V. Krovi, "Orientation workspace and stiffness optimization of cable-driven parallel manipulators with base mobility," ASME Journal of Mechanisms and Robotics, vol. 9, no. 3, p. 031011, 2017

[29] D. Q. Nguyen and M. Gouttefarde, "Study of reconfigurable suspended cable-driven parallel robots for airplane maintenance," in Proc. IEEE/RSJ Int. Conf. Intelligent Robots and Systems (IROS), Chicago, USA, 2014

[30] S. Abdolshah, D. Zanotto, G. Rosati, and S. K. Agrawal, "Optimizing stiffness and dexterity of planar adaptive cable-driven parallel robots," ASME Journal of Mechanisms and Robotics, vol. 9, no. 3, p. 31004 2017.

[31] J.-P. Merlet and D. Daney, "A portable, modular parallel wire crane for rescue operations," in IEEE International Conference on Robotics and Automation (ICRA), Anchorage, Alaska, USA, 2010, pp. 2834-2839.

[32] M. Gouttefarde, S. Krut, O. Company, F. Pierrot, and N. Ramdani, "On the design of fully constrained parallel cable-driven robots," in Advances in Robot Kinematics, J. Lenarčič and P. Wenger, Eds. Springer, 2008, pp. 71-78.

[33] S. Bouchard, C. Gosselin, and B. Moore, "On the ability of a cabledriven robot to generate a prescribed set of wrenches," ASME Journal of Mechanisms and Robotics, vol. 2, no. 1, 2010.

[34] H. Khakpour, L. Birglen, and S.-A. Tahan, "Synthesis of differentially driven planar cable parallel manipulators," IEEE Trans. on Robotics, vol. 30, no. 3, pp. 619-630, Jun. 2014
[35] F. Guay, P. Cardou, A. L. Cruz-Ruiz, and S. Caro, "Measuring how well a structure supports varying external wrenches," in Mechanisms and Machine Science. Springer, 2014, vol. 17, pp. 385-392.

[36] L. Gagliardini, S. Caro, M. Gouttefarde, and A. Girin, "Discrete reconfiguration planning for cable-driven parallel robots," Mechanism and Machine Theory, vol. 100, pp. 313-337, 2016.

[37] L. Gagliardini, S. Caro, M. Gouttefarde, P. Wenger, and A. Girin, "Optimal design of cable-driven parallel robots for large industrial structures," in Proc. IEEE Int. Conf. Robotics and Automation (ICRA), Hong Kong, China, 2014, pp. 5744-5749.

[38] T. Bruckmann, L. Mikelsons, and M. Hiller, "A design-to-task approach for wire robots," in Interdisciplinary Applications of Kinematics. Springer, 2012, pp. 83-97.

[39] J.-P. Merlet, "A generic trajectory verifier for the motion planning of parallel robots," ASME Journal of Mechanical Design, vol. 123, 2001.

[40] F. Hao and J.-P. Merlet, "Multi-criteria optimal design of parallel manipulators based on interval analysis," Mech. and Mach. Theory, vol. 40, no. 2, pp. 157-171, Feb. 2005.

[41] J. T. Bryson, X. Jin, and S. K. Agrawal, "Optimal design of cabledriven manipulators using particle swarm optimization," ASME Journal of Mechanisms and Robotics, vol. 8, no. 4, 2016.

[42] H. Hussein, J. C. Santos, and M. Gouttefarde, "Geometric optimization of a large scale CDPR operating on a building facade," in IEEE/RSJ International Conference on Intelligent Robots and Systems (IROS), 2018, pp. 5117-5124.

[43] M. Hassan and A. Khajepour, "Analysis of bounded cable tensions in cable-actuated parallel manipulators," IEEE Transactions on Robotics, vol. 27, no. 5, pp. 891-900, 2011.

[44] A. Alikhani, S. Behzadipour, A. Alasty, and S. A. S. Vanini, "Design of a large-scale cable-driven robot with translational motion," Robotics and Computer-Integrated Manufacturing, vol. 27, pp. 357-366, 2011.

[45] M. Gouttefarde and S. Krut, "Characterization of parallel manipulator available wrench set facets," in Advances in Robot Kinematics, J. Lenarčič and M. M. Stanišić, Eds. Springer, 2010, pp. 475-482.

[46] M. Hiller, S. Fang, S. Mielczarek, R. Verhoeven, and D. Franitza "Design, analysis and realization of tendon-based parallel manipulators," Mechanism and Machine Theory, vol. 40, no. 4, pp. 429-445, 2005.

[47] G. M. Ziegler, Lectures on polytopes. Springer, 2012, vol. 152.

[48] T. Dallej, M. Gouttefarde, N. Andreff, P.-E. Hervé, and P. Martinet, "Modeling and vision-based control of large-dimension cable-driven parallel robots using a multiple-camera setup," Mechatronics, vol. 61, pp. 20-36, 2019.

[49] K. Fukuda, "Frequently asked questions in polyhedral computation," https://www.cs.mcgill.ca/ fukuda/soft/polyfaq/polyfaq.html, Tech. Rep.

[50] J. Nocedal and S. J. Wright, Numerical Optimization, 2nd ed. Springer, 2006.

[51] ISO 16625:2013(E), "Cranes and hoists: Selection of wire ropes, drums and sheaves."

[52] T. Bruckmann, L. Mikelsons, T. Brandt, M. Hiller, and D. Schramm, "Wire robots part i: Kinematics, analysis and design," in Parallel Manipulators, New Developments, J.-H. Ryu, Ed. I-Tech Education and Publishing, 2008, pp. 109-132.

[53] D. Q. Nguyen and M. Gouttefarde, "Stiffness matrix of 6-dof cabledriven parallel robots and its homogenization," in Advances in Robot Kinematics. Springer, 2014, pp. 181-191.

[54] J. T. Bryson, X. Jin, and S. K. Agrawal, "Configuration robustness analysis of the optimal design of cable-driven manipulators," ASME Journal of Mechanisms and Robotics, vol. 8, no. 6, 2016.

[55] M. Gouttefarde, "Characterizations of fully constrained poses of parallel cable-driven robots: A review," in Proc. ASME Int. Design Engineering Technical Conf. and Computers and Information in Engineering Conf., no. DETC2008-49467, Brooklyn, NY, 2008.

\section{APPENDIX A \\ PROOF OF (14)}

Consider the optimization problem (13):

$$
\begin{aligned}
& \min _{\mathbf{t}_{\max }}\left\|\mathbf{t}_{\max }\right\|_{\infty} \\
& \text { subject to } \quad \mathbf{A t}_{\max } \geq \mathbf{b}
\end{aligned}
$$

where $\mathbf{A}=\left[\mathbf{a}_{1}^{T}, \ldots, \mathbf{a}_{q}^{T}\right]^{T}, \mathbf{b}=\left[b_{1}, \ldots, b_{q}\right]^{T}, a_{j i} \geq 0 \forall i, j$, and $\mathbf{A t}_{\max } \geq \mathbf{b}$ is assumed consistent (there exists at least one $\mathbf{x} \in \mathbb{R}^{m}$ such that $\mathbf{A x} \geq \mathbf{b}$; i.e., $b_{j} \leq 0$ if $\mathbf{a}_{j}=\mathbf{0}$ ). 
An optimal solution to this problem is given by:

$$
\mathbf{t}_{\max }^{*}=\max _{j \in J_{+}}\left(\frac{b_{j}}{\sum_{i=1}^{m} a_{j i}}\right) \mathbf{1}_{m}
$$

where $\mathbf{1}_{m}=[1,1, \ldots, 1]^{T} \in \mathbb{R}^{m}$ and $J_{+}=\left\{j \mid \sum_{i=1}^{m} a_{j i}>0\right\}$, $J_{+} \subseteq\{1,2, \ldots, q\}$.

Proof: First, note that $\mathbf{t}_{\max }^{*}$ satisfies the problem constraints $\mathbf{A t _ { \text { max } }} \geq \mathbf{b}$ since, for any $j \in J_{+}$:

$$
\begin{aligned}
\mathbf{a}_{j} \mathbf{t}_{\max }^{*} & =\max _{j \in J_{+}}\left(\frac{b_{j}}{\sum_{i=1}^{m} a_{j i}}\right) \mathbf{a}_{j} \mathbf{1}_{m}=\max _{j \in J_{+}}\left(\frac{b_{j}}{\sum_{i=1}^{m} a_{j i}}\right) \sum_{i=1}^{m} a_{j i} \\
& \geq \frac{b_{j}}{\sum_{i=1}^{m} a_{j i}} \sum_{i=1}^{m} a_{j i}=b_{j} .
\end{aligned}
$$

Moreover, by contradiction, assume that there is a vector $\mathbf{x} \in \mathbb{R}^{m}, \mathbf{x}=\left[x_{1}, \ldots, x_{m}\right]^{T}$ verifying the following two conditions:

$$
\begin{aligned}
\mathbf{A x} & \geq \mathbf{b} \\
\|\mathbf{x}\|_{\infty}<\left\|\mathbf{t}_{\max }^{*}\right\|_{\infty} & \Longleftrightarrow \max _{i}\left(x_{i}\right)<\max _{j \in J_{+}}\left(\frac{b_{j}}{\sum_{i=1}^{m} a_{j i}}\right)
\end{aligned}
$$

Let $h=\underset{j \in J_{+}}{\operatorname{argmax}}\left(\frac{b_{j}}{\sum_{i=1}^{m} a_{j i}}\right)$, then (49) implies $\max _{i}\left(x_{i}\right)<\frac{b_{h}}{\sum_{i=1}^{m} a_{h i}}$ and hence $x_{i}<\frac{b_{h}}{\sum_{i=1}^{m} a_{h i}}, \forall i$. Consequently, since $a_{h i}>0$ for some $i$ and $a_{h i}=0$ for all other $i$ (because $h \in J_{+}$and $a_{j i} \geq 0$, $\forall i, j)$, we have $\sum_{i=1}^{m} a_{h i}=\sum_{a_{h i}>0} a_{h i}$ and then:

$$
\mathbf{a}_{h} \mathbf{x}=\sum_{i=1}^{m} a_{h i} x_{i}=\sum_{a_{h i}>0} a_{h i} x_{i}<\sum_{a_{h i}>0} a_{h i} \frac{b_{h}}{\sum_{i=1}^{m} a_{h i}}=b_{h}
$$

i.e., $\mathbf{a}_{h} \mathbf{x}<b_{h}$ which contradicts (48). Therefore, $\forall \mathbf{x}$ such that $\mathbf{A x} \geq \mathbf{b},\|\mathbf{x}\|_{\infty} \geq\left\|\mathbf{t}_{\max }^{*}\right\|_{\infty}$, i.e., $\mathbf{t}_{\max }^{*}$ is an optimal solution to the optimization problem (13).

Note that this solution is generally not unique as discussed in Section III-C.

Furthermore, let us prove the following result stated in Section III-A: When $\operatorname{rank}(\mathbf{W})=n, \bigcup_{j} I_{j}^{+}=\{1, \ldots, m\}$.

Proof: Consider any $i \in\{1, \ldots, m\}$. Since $\operatorname{rank}(\mathbf{W})=n$, there exists $n-1$ linearly independent columns $\mathbf{w}_{i_{1}}, \quad \mathbf{w}_{i_{2}}, \ldots, \quad \mathbf{w}_{i_{n-1}}$ of the wrench matrix $\mathbf{W}$ such that $\operatorname{rank}\left(\left[\mathbf{w}_{i}, \mathbf{w}_{i_{1}}, \mathbf{w}_{i_{2}}, \ldots, \mathbf{w}_{i_{n-1}}\right]\right)=n$. Let us define $I=\left\{i_{1}, \ldots, i_{n-1}\right\}$ and $\mathbf{M}_{I}=\left[\mathbf{w}_{i_{1}}, \mathbf{w}_{i_{2}}, \ldots, \mathbf{w}_{i_{n-1}}\right]^{T}$. According to (5), there exists two rows of $\mathbf{C}, \mathbf{c}_{k}$ and $\mathbf{c}_{l}$, such that $\mathbf{c}_{k}=-\mathbf{c}_{l}=\operatorname{null}\left(\mathbf{M}_{I}\right)^{T}$. If $\mathbf{c}_{k} \mathbf{w}_{i}=\mathbf{c}_{l} \mathbf{w}_{i}=0$, it means that $\mathbf{w}_{i}$ is orthogonal to null $\left(\mathbf{M}_{I}\right)$, i.e., that $\mathbf{w}_{i} \in \operatorname{span}\left(\mathbf{w}_{i_{1}}, \mathbf{w}_{i_{2}}, \ldots, \mathbf{w}_{i_{n-1}}\right)$ which contradicts $\operatorname{rank}\left(\left[\mathbf{w}_{i}, \mathbf{w}_{i_{1}}, \mathbf{w}_{i_{2}}, \ldots, \mathbf{w}_{i_{n-1}}\right]\right)=n$. Hence, $\mathbf{c}_{k} \mathbf{w}_{i} \neq 0$ and $\mathbf{c}_{l} \mathbf{w}_{i} \neq 0$ which implies that $i \in I_{k}^{+}$or $i \in I_{l}^{+}$ $\left(i \in I_{k}^{+}\right.$if $\mathbf{c}_{k} \mathbf{w}_{i}>0$ and $i \in I_{l}^{+}$otherwise since $\mathbf{c}_{k} \mathbf{w}_{i}<0$ implies $\mathbf{c}_{l} \mathbf{w}_{i}>0$ because $\mathbf{c}_{k}=-\mathbf{c}_{l}$ ). We just proved that for any $i \in\{1, \ldots, m\}$, there exists $j$ such that $i \in I_{j}^{+}$and hence $\bigcup_{j} I_{j}^{+}=\{1, \ldots, m\}$.

\section{APPENDIX B}

\section{A NECESSARY AND SUFFICIENT CONDITION FOR $I_{j}^{+} \neq \emptyset$}

A pose of the mobile platform of a CDPR belongs to the WCW if any wrench $\mathbf{f}$ can be produced at the platform by pulling on it with the non-negative cable tensions, i.e.:

$$
\forall \mathbf{f} \in \mathbb{R}^{n}, \exists \mathbf{t} \geq \mathbf{0} \in \mathbb{R}^{m} \text { such that } \mathbf{W t}=\mathbf{f} .
$$

Well-known necessary conditions for the WCW to exist are $\operatorname{rank}(\mathbf{W})=n$ (full rank) and $m \geq n+1$ (more cables than DOFs). Furthermore, the poses belonging to the WCW can be characterized by means of the following theorem [16], [55].

Theorem Assuming that $\operatorname{rank}(\mathbf{W})=n$, a pose of the mobile platform belongs to the WCW if and only if all the hyperplanes $\mathscr{H}$ of $\mathbb{R}^{n}$ spanned by $n-1$ linearly independent columns of the wrench matrix $\mathbf{W}$ are separating, i.e.,

$$
\exists(i, k), i \neq k, \text { such that } \mathbf{q}^{T} \mathbf{w}_{i}>0 \text { and } \mathbf{q}^{T} \mathbf{w}_{k}<0
$$

where $\mathbf{q} \neq \mathbf{0}$ is a vector orthogonal to $\mathscr{H}$.

Based on this Theorem, the following result is proved below: $I_{j}^{+} \neq \emptyset \forall j, 1 \leq j \leq p$, if and only if the pose of the CDPR mobile platform belongs to the wrench-closure workspace (WCW).

Proof: By definition, $I_{j}^{+}=\left\{i \mid \mathbf{c}_{j} \mathbf{w}_{i}>0\right\}$ and according to its definition in (5), $\mathbf{c}_{j}$ is orthogonal to a hyperplane spanned by $n-1$ columns of $\mathbf{W}$. Consequently, if a pose belongs to the WCW, the Theorem above implies that there exists $i$ such that $\mathbf{c}_{j} \mathbf{w}_{i}>0$ for all $j, 1 \leq j \leq p$ (choosing $\mathbf{q}=\mathbf{c}_{j}^{T}$ in (52)), i.e., that $I_{j}^{+} \neq \emptyset \forall j$ which proves the "if" part. Now, to prove the "only if" part, assume that $I_{j}^{+} \neq \emptyset \forall j, 1 \leq j \leq p$, and consider any hyperplane $\mathscr{H}$ spanned by $n-1$ linearly independent columns of $\mathbf{W}$. By definition of matrix $\mathbf{C}$ (Section II-B), there is a row $\mathbf{c}_{j}$ of $\mathbf{C}$ orthogonal to $\mathscr{H}$. Since $I_{j}^{+} \neq \emptyset$, there exists $i$ such that $\mathbf{c}_{j} \mathbf{w}_{i}>0$ (note that this implies that $\operatorname{rank}(\mathbf{W})=n$ ). Moreover, according to (5), there is a row $\mathbf{c}_{l}$ in $\mathbf{C}$ such that $\mathbf{c}_{l}=-\mathbf{c}_{j}$. Since $I_{l}^{+} \neq \emptyset$, there exists $k$ such that $\mathbf{c}_{l} \mathbf{w}_{k}>0$ and hence $\mathbf{c}_{j} \mathbf{w}_{k}<0$. Since $\mathbf{c}_{j} \mathbf{w}_{i}>0$ and $\mathbf{c}_{j} \mathbf{w}_{k}<0$, the hyperplane $\mathscr{H}$ is separating. $\mathscr{H}$ being any hyperplane spanned by $n-1$ linearly independent columns of $\mathbf{W}$, the Theorem above implies that the pose of the mobile platform belongs to the WCW.

\section{APPENDIX C \\ ProOF OF THE NATURE OF $\mathbb{T}_{\text {MAX }}$}

Let $\mathbb{T}_{\max }$ be the set of all optimal solutions to (13). This appendix proves that $\mathbb{T}_{\max }$ can be represented as the solution set of a system of linear inequalities. As a consequence, $\mathbb{T}_{\max }$ is a convex polytope.

Since $\mathbf{t}_{\max }^{*}$ defined in (14) belongs to $\mathbb{T}_{\max }$, any other $\mathbf{t}_{\max }$ in $\mathbb{T}_{\max }$ shall verify $\left\|\mathbf{t}_{\max }\right\|_{\infty}=t_{\max }^{*}$. Hence, $\mathbb{T}_{\max }$ lies in $\mathbb{R}^{m}$ on a face of the infinity norm level set (a hypercube) of value $t_{\max }^{*}$. More precisely, let us first prove that:

$$
\mathbf{t}_{\max } \in \mathbb{T}_{\max } \quad \Longrightarrow \quad t_{\max , i}=t_{\max }^{*}, \forall i \in I_{h}^{+}
$$

where $h$ is defined in (17) and, referring to Sections II-B and III-A, $I_{h}^{+}=\left\{i \mid \mathbf{c}_{h} \mathbf{w}_{i}>0\right\}=\left\{i \mid a_{h i}>0\right\}$.

Proof: Any $\mathbf{t}_{\max } \in \mathbb{T}_{\max }$ is such that $\mathbf{A t}_{\max } \geq \mathbf{b}$ and $\left\|\mathbf{t}_{\max }\right\|_{\infty}=t_{\max }^{*}$. The latter implies that $t_{\max , i} \leq t_{\max }^{*}$ for all $i$. Assume that $\exists i \in I_{h}^{+}$such that $t_{\max , i}<t_{\max }^{*}$. By definition of $h$, we have $t_{\max }^{*}=\frac{b_{h}}{\sum_{a_{h i}>0} a_{h i}}=\frac{b_{h}}{\sum_{i \in I_{h}^{+}} a_{h i}}$. Then, similarly to (50) in Appendix A, we can write:

$$
\mathbf{a}_{h} \mathbf{t}_{\max }=\sum_{a_{h i}>0} a_{h i} t_{\max , i}<t_{\max }^{*} \sum_{a_{h i}>0} a_{h i}=b_{h}
$$

which contradicts the fact that $\mathbf{A t}_{\max } \geq \mathbf{b}$ and thus proves that $t_{\max , i}=t_{\max }^{*}, \forall i \in I_{h}^{+}$. 
Note that for each $i \in I_{h}^{+}, t_{\max , i}=t_{\max }^{*}$ defines an hyperplane in the space of the maximum cable tensions $\mathbf{t}_{\max }$. Then, (53) shows that $\mathbb{T}_{\max }$ lies on the intersection of all these hyperplanes.

Now, let us prove that the set $\mathbb{T}_{\text {max }}$ of all optimal solutions $\mathbf{t}_{\text {max }}$ to (13) corresponds to the solution set of the following system of linear inequalities (and is thus a convex polytope):

$$
\begin{gathered}
t_{\max , i}=t_{\max }^{*}, \forall i \in I_{h}^{+} \\
t_{\max , i} \leq t_{\max }^{*}, \forall i \notin I_{h}^{+} \\
\mathbf{A t}_{\max } \geq \mathbf{b}
\end{gathered}
$$

where $h$ is defined in (17) and $t_{\max }^{*}$ is defined in (15).

Proof: On the one hand, if $\mathbf{t}_{\max }$ verifies (55), (56) and (57), its infinity norm is equal to $t_{\max }^{*}$ and $\mathbf{A} \mathbf{t}_{\max } \geq \mathbf{b}$. Hence, $\mathbf{t}_{\max } \in \mathbb{T}_{\max }$, i.e., it is an optimal solution to (13). On the other hand, if $\mathbf{t}_{\max } \in \mathbb{T}_{\max }$ then (56) is satisfied since $\left\|\mathbf{t}_{\max }\right\|_{\infty}=t_{\max }^{*}$ and (57) is true since $\mathbf{t}_{\max }$ is a solution to (13). Moreover, (55) is true according to (53).

Note also that (55) and (56) indicate that all the optimal solutions in $\mathbb{T}_{\max }$ have maximum cable tension components smaller or equal to those of $\mathbf{t}_{\max }^{*}$ (i.e., to $t_{\max }^{*}$ ), which shall be expected from the outset from the formulation of (13) where the minimum of the infinity norm is used.

\section{APPENDIX D}

\section{Calculation of $\mathbf{t}_{\text {MAX }}^{m}$ AND Algorithm 1}

At the first iteration of the while loop in Algorithm 1, $\mathbf{A}^{r}=\mathbf{A}, \mathbf{b}^{r}=\mathbf{b}$ and $I_{\text {removed }}=\{\emptyset\}$ so that the components of $\mathbf{t}_{\max }^{m}$ of indices $i \in I_{h}^{+}=\left\{i \mid a_{h i}>0\right\}$ are calculated as being equal to $t_{\max }^{*}$ given in (15). Then, $\mathbf{A}^{r}$ is obtained from $\mathbf{A}$ by removing all its columns of indices $i \in I_{h}^{+}\left(\mathbf{A}^{r} \in \mathbb{R}^{q \times(m-z)}\right.$ where $\left.z=\operatorname{card}\left(I_{h}^{+}\right)\right)$and the components $b_{j}^{r}$ of $\mathbf{b}^{r}$ are defined as $b_{j}^{r}=b_{j}-\sum_{i \in I_{h}^{+}} a_{j i} t_{\max }^{*}$. Let us prove that the set $\mathbb{T}_{\text {max }}$ of all optimal solutions $\mathbf{t}_{\text {max }}$ to (13) corresponds to the solution set of the following system of linear inequalities:

$$
\begin{gathered}
t_{\max , i}=t_{\max }^{*}, \forall i \in I_{h}^{+} \\
t_{\max , i} \leq t_{\max }^{*}, \forall i \notin I_{h}^{+} \\
\mathbf{A}^{r} \mathbf{t}_{\max }^{r} \geq \mathbf{b}^{r}
\end{gathered}
$$

where $\mathbf{t}_{\max }^{r}$ is obtained from $\mathbf{t}_{\max }$ by removing all components of index $i \in I_{h}^{+}$, i.e., $\mathbf{t}_{\max }^{r} \in \mathbb{R}^{m-z}$ is composed of the components $t_{\max , i}$ for all $i \notin I_{h}^{+}$. In words, the inequality system (60) is obtained from $\mathbf{A t}_{\max } \geq \mathbf{b}$ as follows: For all $i \in I_{h}^{+}$and all $j, 1 \leq j \leq q$, move $a_{j i} t_{\max , i}$ to the right-hand side of the inequality $\mathbf{a}_{j} \mathbf{t}_{\max } \geq b_{j}$ and set $t_{\max , i}=t_{\max }^{*}$.

Proof: On the one hand, if $\mathbf{t}_{\max }$ verifies (58), (59) and (60), its infinity norm is equal to $t_{\max }^{*}$ and $\mathbf{A} \mathbf{t}_{\max } \geq \mathbf{b}$ by definition of $\mathbf{A}^{r}$, $\mathbf{t}_{\max }^{r}$ and $\mathbf{b}^{r}$. Hence, $\mathbf{t}_{\max }$ is an optimal solution to (13) $\left(\mathbf{t}_{\max } \in \mathbb{T}_{\max }\right)$. On the other hand, if $\mathbf{t}_{\max } \in \mathbb{T}_{\max }$ then (59) is satisfied since $\left\|\mathbf{t}_{\max }\right\|_{\infty}=t_{\text {max }}^{*}$. Moreover, (58) is true according to (53) and, as a consequence, $\mathbf{A} \mathbf{t}_{\max } \geq \mathbf{b}$ implies that $\mathbf{A}^{r} \mathbf{t}_{\max }^{r} \geq \mathbf{b}^{r}$. Hence, $\mathbf{t}_{\max }$ verifies (58), (59) and (60).

Now, our goal is to minimize the maximum of the components $t_{\max , i}, i \notin I_{h}^{+}$, of $\mathbf{t}_{\max }$ while keeping the resulting vector inside $\mathbb{T}_{\max }$. Since the components $t_{\max , i}, i \notin I_{h}^{+}$, are those of $\mathbf{t}_{\max }^{r}$ and according to (60), this minimization problem can be written as:

$$
\begin{gathered}
\min _{\mathbf{t}_{\max }^{r}}\left\|\mathbf{t}_{\max }^{r}\right\|_{\infty} \\
\text { subject to } \quad \mathbf{A}^{r} \mathbf{t}_{\max }^{r} \geq \mathbf{b}^{r} .
\end{gathered}
$$

According to Section III-A, the optimal value of this problem, denoted here as $t_{\max }^{r, *}$, is obtained by using (15) with $\mathbf{A}^{r}$ and $\mathbf{b}^{r}$ in place of $\mathbf{A}$ and $\mathbf{b}$. Hence, $t_{\max }^{r, *}$ is obtained from row $h^{r}$ of $\mathbf{A}^{r} \mathbf{t}_{\max }^{r} \geq \mathbf{b}^{r}$, with $h^{r}$ defined as (cf. (17)):

$$
h^{r}=\underset{j \in J_{+}^{r}}{\operatorname{argmax}}\left(\frac{b_{j}^{r}}{\sum_{i} a_{j i}^{r}}\right)
$$

where $a_{j i}^{r}$ are the components of matrix $\mathbf{A}^{r}$. Row $h^{r}$ of $\mathbf{A}^{r} \mathbf{t}_{\max }^{r} \geq \mathbf{b}^{r}$ only involves the components of $\mathbf{t}_{\max }^{r}$ of indices $i$ such that $a_{h^{r} i}^{r}>0$. Hence, to obtain an optimal solution to (61), we set $t_{\max , i}^{r}=t_{\max }^{r, *}$ for $i \in\left\{i \mid a_{h^{r} i}^{r}>0\right\}$. For the moment, we leave free the other components of $\mathbf{t}_{\max }^{r}$ in order to be able to further minimize them in the next steps of the method (next iterations of the while loop in Algorithm 1). Then, the while loop in Algorithm 1 ends with the computations of the new $\mathbf{A}^{r}$ and $\mathbf{b}^{r}$ which are computed from the current $\mathbf{A}^{r}$ and $\mathbf{b}^{r}$ as the latter were computed from $\mathbf{A}$ and $\mathbf{b}$. In the next while loop, the minimization problem (61) is solved again for the new $\mathbf{A}^{r}$ and $\mathbf{b}^{r}$ and this process continues until all components of $\mathbf{t}_{\max }^{m}$ have been set, $I_{\text {removed }}=\{1, \ldots, m\}$ and line 13 of Algorithm 1 results in $\mathbf{A}^{r}$ being empty.

Note that the manipulations on $I_{\text {removed }}$ and $I_{h}^{+}$at lines 7,8 and 12 of Algorithm 1 are used to keep track of the indices of the components $t_{\max , i}^{m}$ of $\mathbf{t}_{\max }^{m}$ which must be set to $t_{\max }^{r, *}$ at each iteration of the while loop (at line 9). Notably, $I_{h}^{+}$is calculated at line 7 from row $h$ of matrix $\mathbf{A}$ and not from matrix $\mathbf{A}^{r}$ for that purpose. Besides, $h$ is used in place of $h^{r}$ in Algorithm 1 for simplicity of notation.

The principle of the computation of $\mathbf{t}_{\max }^{m}$ proposed in Section III-C and its implementation in Algorithm 1 have been outlined above. However, it remains to be proved that this method is correct, i.e., that $\mathbf{t}_{\max }^{m} \in \mathbb{T}_{\max }$, that the components $t_{\max , i}^{m}$ of $\mathbf{t}_{\max }^{m}$, which are successively computed in the method (line 9 in Algorithm 1), are smaller and smaller, and that the while loop in Algorithm 1 terminates in a finite number of steps (in fact, in a maximum of $m$ steps).

First, let us prove that $t_{\max }^{r * *} \leq t_{\max }^{*}$ where:

$$
t_{\max }^{r, *}=\max _{j \in J_{+}^{r}}\left(\frac{b_{j}^{r}}{\sum_{i} a_{j i}^{r}}\right)=\frac{b_{h^{r}}^{r}}{\sum_{i} a_{h^{r} i}^{r}} .
$$

$J_{+}^{r}=\left\{j \mid \sum_{i} a_{j i}^{r}>0\right\}, h^{r}$ is given by (62), and $t_{\max }^{*}$ is given by (15), i.e., prove that the optimal value of problem (61) is smaller than the one of problem (13). This allows one to straightforwardly prove by induction that the components $t_{\text {max }, i}^{m}$ of $\mathbf{t}_{\max }^{m}$, which are successively computed in the while loop at line 9 in Algorithm 1, are smaller and smaller, all of them being less than or equal to $t_{\max }^{*}$.

Proof of $t_{\text {max }}^{r * *} \leq t_{\text {max }}^{*}$ : By definition of $\mathbf{b}^{r}$, we have $b_{h^{r}}^{r}=b_{h^{r}}-t_{\max }^{*} \sum_{i \in I_{h}^{+}} a_{h^{r} i}$ and then, according to (63):

$$
t_{\max }^{r, *}=\frac{b_{h^{r}}-t_{\max }^{*} \sum_{i \in I_{h}^{+}} a_{h^{r} i}}{\sum_{i} a_{h^{r} i}^{r}} .
$$


Moreover:

$$
\sum_{i} a_{h^{r} i}^{r}=\sum_{i \in I_{h^{r}}^{r,+}} a_{h^{r}}^{r}
$$

where $I_{h^{r}}^{r,+}=\left\{i \mid a_{h^{r} i}^{r}>0\right\}$ (sorry for the cumbersome notations). Now, the crucial observation is that:

$$
\sum_{i \in I_{h^{r}}^{+}} a_{h^{r} i}=\sum_{i \in I_{h^{r}}^{r,+}} a_{h^{r} i}^{r}+\sum_{i \in I_{h}^{+}} a_{h^{r}}
$$

where by definition $I_{h^{r}}^{+}=\left\{i \mid a_{h^{r} i}>0\right\}$ is the index set of all the strictly positive components of row $h^{r}$ of A. Eq. (66) is true since $\mathbf{A}^{r}$ has been obtained from $\mathbf{A}$ by removing all columns of $\mathbf{A}$ of indices $i \in I_{h}^{+}$. Hence, the components $a_{h^{r} i}^{r}>0$ of row $h^{r}$ of $\mathbf{A}^{r}$ correspond to some of the components $a_{h^{r} i}>0$ of row $h^{r}$ of $\mathbf{A}$ and the indices $i$ of the $a_{h^{r}}>0$ which are not in row $h^{r}$ of $\mathbf{A}^{r}$ belong necessarily to $I_{h}^{+}$, which proves (66). But, by definition of $t_{\max }^{*}$ in (15), we have:

$$
t_{\max }^{*} \geq \frac{b_{h^{r}}}{\sum_{i \in I_{h^{r}}^{+}} a_{h^{r}}}
$$

and hence, using (66):

$$
\left(\sum_{i \in I_{h^{r}}^{+}} a_{h^{r}}\right) t_{\max }^{*}=\left(\sum_{i \in I_{h^{r}}^{r,+}} a_{h^{r} i}^{r}+\sum_{i \in I_{h}^{+}} a_{h^{r} i}\right) t_{\max }^{*} \geq b_{h^{r}}
$$

i.e.,

$$
t_{\max }^{*} \geq \frac{b_{h^{r}}-t_{\max }^{*} \sum_{i \in I_{h}^{+}} a_{h^{r} i}}{\sum_{i \in I_{h^{r}}^{r+}} a_{h^{r} i}^{r}} .
$$

According to (64) and (65), the right-hand side of (69) is equal to $t_{\text {max }}^{r, *}$ which proves that $t_{\max }^{*} \geq t_{\text {max }}^{r, *}$.

Note that it is not difficult to figure out that $t_{\max }^{*} \geq 0$ and $t_{\max , i}^{m} \geq t_{\min , i}$ for all $i$ since the last $n$ rows of $\mathbf{A} \mathbf{t}_{\max } \geq \mathbf{b}$ in (9) were defined to enforce $\mathbf{t}_{\max } \geq \mathbf{t}_{\min } \geq \mathbf{0}$.

Now, let us prove that Algorithm 1 terminates in a finite number of steps, this number being less than or equal to $m$.

Proof: It suffices to prove that, from one iteration to the next one of the while loop in Algorithm 1, the number of columns of $\mathbf{A}^{r}$ is decreasing. It amounts to show that $I_{h^{r}}^{+}$, calculated at line 8 in Algorithm 1, is not empty ( $h$ is used in place of $h^{r}$ in Algorithm 1 for simplicity of notation). $I_{h^{r}}^{+}$is the index set of the strictly positive components of row $h^{r}$ of $\mathbf{A}^{r}$, where $h^{r}$ is obtained at line 6. According to (62), $I_{h^{r}}^{+}$is empty if and only if $J_{+}^{r}=\left\{j \mid \sum_{i} a_{j i}^{r}>0\right\}$ is empty which is not possible because of the last $m$ rows of $\mathbf{A t}_{\max } \geq \mathbf{b}$ in (9). Indeed, these last $m$ rows form the identity matrix and, since $\mathbf{A}^{r}$ has been obtained at line 13 of the previous iteration by removing some of columns of $\mathbf{A}$ (but not all its columns since otherwise the algorithm would have already been terminated), it ensures that there exists at least one row of $\mathbf{A}^{r}$ with at least one $a_{j i}^{r}>0$, i.e., that $J_{+}^{r}$ is not empty. As a consequence, the index of at least one new column of $\mathbf{A}$ is added to $I_{\text {removed }}$ at each iteration of the while loop in Algorithm 1 which shows that the latter terminates in a maximum of $m$ steps.

Finally, to prove that $\mathbf{t}_{\text {max }}^{m}$ belongs to $\mathbb{T}_{\text {max }}$, it suffices to prove that $\mathbf{t}_{\max }^{m}$ satisfies (55), (56) and (57) (see Appendix C).

Proof: First, referring to the calculations made at the first iteration of the while loop in Algorithm 1, (55) is satisfied by $\mathbf{t}_{\max }^{m}$ by construction. Moreover, the components $t_{\max , i}^{m}$ satisfies
(56) for all $i \notin I_{h}^{+}$since we proved above that the $t_{\max , i}^{m}$ which are successively computed in the while loop in Algorithm 1, are smaller and smaller, all of them being less than or equal to $t_{\max }^{*}$. Finally, to see that $\mathbf{t}_{\max }^{m}$ satisfies (57), it suffices to note that at the end of Algorithm 1, at line 11, we have for all $j$ :

$0 \geq b_{j}-\sum_{(0) I_{h}^{+}} a_{j i}{ }^{(0)} t_{\max }^{*}-\sum_{(1) I_{h}^{+}} a_{j i}{ }^{(1)} t_{\max }^{*}-\ldots-\sum_{(s)} I_{h}^{+} a_{j i}{ }^{(s)} t_{\max }^{*}$

where $s$ is the total number of iterations $(s \leq m)$, and ${ }^{(i)} t_{\max }^{*}$ and ${ }^{(i)} I_{h}^{+}$are $t_{\max }^{r, *}$ and $I_{h}^{+}$calculated respectively at lines 5 and 8 at the $i$-th iteration of the while loop in Algorithm 1. The righthand side of (70) is less than or equal to zero by definition of ${ }^{(s)} t_{\max }^{*}$ (Eq. (15) applied to the matrix $\mathbf{A}^{r}$ and vector $\mathbf{b}^{r}$ obtained in the end of the second to last iteration of the while loop). By construction of $\mathbf{t}_{\max }^{m}$ (line 9 in Algorithm 1), its components of indices in ${ }^{(i)} I_{h}^{+}$are set equal to ${ }^{(i)} t_{\max }^{*}$ at the $i$-th iteration. Hence, (70) is equivalent to $\mathbf{0} \geq \mathbf{b}-\mathbf{A t}_{\text {max }}^{m}$, i.e., $\mathbf{t}_{\max }^{m}$ satisfies (57).

\section{APPENDIX E}

PROOF OF (19)

As stated in Section III-C, the set $\mathbb{T}_{\max }$ of all optimal solutions to (13) is a convex polytope. In this appendix, it is proved that the box:

$$
\mathbb{B}=\left\{\mathbf{t}_{\max } \mid t_{\max , i}^{m} \leq t_{\max , i} \leq t_{\max }^{*}, i=1 \ldots m\right\}
$$

is included into $\mathbb{T}_{\max }\left(\mathbb{B} \subseteq \mathbb{T}_{\max }\right)$, where $\mathbf{t}_{\max }^{m}$ and $\mathbf{t}_{\text {max }}^{*}$ are two particular optimal solutions to (13) defined in Section III-C.

Proof: Consider $\mathbf{t}_{\max } \in \mathbb{B}$. The inequality constraints $\mathbf{A t}_{\max } \geq \mathbf{b}$ are satisfied since $\mathbf{t}_{\max } \geq \mathbf{t}_{\max }^{m}$ according to (71) and, all components $a_{j i}$ of $\mathbf{A}$ being non-negative, we have $\mathbf{A t _ { \operatorname { m a x } }} \geq \mathbf{A} \mathbf{t}_{\max }^{m} \geq \mathbf{b}$. Moreover, there exists at least one $i$ such that $t_{\max , i}^{m}=t_{\max }^{*}(i=h$ with $h$ defined in (17)). According to (71), we then have $\left\|\mathbf{t}_{\max }\right\|_{\infty}=t_{\max }^{*}$, i.e., $\mathbf{t}_{\max }$ belongs to $\mathbb{T}_{\max }$ (it is an optimal solution to (13)).

It may be true that $\mathbb{T}_{\max }=\mathbb{B}$ (we did not find a counter example) but we did not have a proof of this result.

\section{APPENDIX F \\ PROOF OF (24)}

The proof of (24) is similar to the one of (14) in Appendix A and it is thus only sketched. First, note that $\mathbf{A t}_{\max } \geq \mathbf{b}$ is assumed to be consistent for all $\mathbf{f} \in \mathrm{RW}$, i.e., we assume that, for all $\mathbf{f} \in \mathrm{RW}, b_{j}=b_{j}(\mathbf{f}) \leq 0$ for all $j$ such that $\mathbf{a}_{j}=\mathbf{0}$ (otherwise, the current pose if outside the wrenchfeasible workspace whatever $\left.\mathbf{t}_{\max }\right)$. Then, $\mathbf{t}_{\max , \mathrm{RW}}^{*}$ satisfies the constraints of (23) for any $\mathbf{f} \in \mathrm{RW}$ since, for any $j \in J_{+}$:

$$
\begin{aligned}
\mathbf{a}_{j} \mathbf{t}_{\text {max }, \mathrm{RW}}^{*} & =\max _{j \in J_{+}}\left(\frac{\max _{\mathbf{f} \in \mathrm{RW}} b_{j}}{\sum_{i=1}^{m} a_{j i}}\right) \mathbf{a}_{j} \mathbf{1}_{m}=\max _{j \in J_{+}}\left(\frac{\max _{\mathbf{f} \in \mathrm{RW}} b_{j}}{\sum_{i=1}^{m} a_{j i}}\right) \sum_{i=1}^{m} a_{j i} \\
& \geq \frac{\max _{\mathbf{f} \in \mathrm{RW}} b_{j}}{\sum_{i=1}^{m} a_{j i}} \sum_{i=1}^{m} a_{j i}=\max _{\mathbf{f} \in \mathrm{RW}} b_{j} \geq b_{j}
\end{aligned}
$$

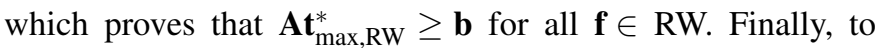
prove that $\mathbf{t}_{\text {max,RW }}^{*}$ is an optimal solution to (23) (and thus that 
$t_{\text {max, RW }}^{*}$ defined in (24) is the optimal value of (23)), assume to the contrary that there exists a vector $\mathbf{t}_{\max }$ such that:

$$
\begin{array}{r}
\mathbf{A t}_{\max } \geq \mathbf{b}, \forall \mathbf{f} \in \mathrm{RW} \\
\left\|\mathbf{t}_{\text {max }}\right\|_{\infty}<\left\|\mathbf{t}_{\text {max }, \mathrm{RW}}^{*}\right\|_{\infty}=t_{\text {max }, \mathrm{RW}}^{*} .
\end{array}
$$

Let index $h$ be defined as:

$$
h=\underset{j \in J_{+}}{\operatorname{argmax}}\left(\frac{\max _{\mathbf{f} \in \mathrm{RW}} b_{j}}{\sum_{i} a_{j i}}\right) .
$$

Then (73) implies $\max _{i}\left(t_{\max , i}\right)<\frac{\max _{\mathbf{f} \in \mathrm{RW}} b_{h}}{\sum_{i} a_{h i}}$. Consequently, since $a_{h i} \geq 0$ for all $i$ and $a_{h i}>0$ for some $i$ (since $h \in J_{+}$), we have:

$$
\mathbf{a}_{h} \mathbf{t}_{\max }=\sum_{i} a_{h i} t_{\max , i}<\sum_{i} a_{h i} \frac{\max _{\mathbf{f} \in \mathrm{RW}} b_{h}}{\sum_{i} a_{h i}}=\max _{\mathbf{f} \in \mathrm{RW}} b_{h} .
$$

With $\mathbf{f}^{*}=\underset{\mathbf{f} \in \mathrm{RW}}{\operatorname{argmax}} b_{h}$ and $b_{h}^{*}=b_{h}\left(\mathbf{f}^{*}\right)=\max _{\mathbf{f} \in \mathrm{RW}} b_{h}$, (75) is $\mathbf{a}_{h} \mathbf{t}_{\max }<b_{h}^{*}$ which implies that (72) is false since $\mathbf{f}^{*} \in \mathrm{RW}$, a contradiction.

This proof illustrates basically that replacing $b_{j}$ by $\max _{\mathbf{f} \in \mathrm{RW}} b_{j}$ and $\mathbf{A t}_{\max } \geq \mathbf{b}$ by $\mathbf{A t}_{\text {max }} \geq \mathbf{b}, \forall \mathbf{f} \in \mathrm{RW}$ allow us to generalize (14) to (24), i.e., to generalize to a set of wrenches RW. This generalization is simple since matrix $\mathbf{A}$ is independent of the wrench $\mathbf{f}$.

\section{APPENDIX G}

\section{PROOF OF THE NATURE OF $\mathbb{T}_{\text {MAX,RW }}$}

Let $\mathbb{T}_{\text {max,RW }}$ be the set of all optimal solutions to (23). This appendix proves that $\mathbb{T}_{\max , \mathrm{RW}}$ is the solution set of a system of linear inequalities and is thus a convex polytope. The proof is very similar to the one presented in Appendix C.

First, let us prove that:

$$
\mathbf{t}_{\max } \in \mathbb{T}_{\max , \mathrm{RW}} \quad \Longrightarrow \quad t_{\max , i}=t_{\max , \mathrm{RW}}^{*}, \forall i \in I_{h}^{+}
$$

where $h$ is defined in (74) and $I_{h}^{+}=\left\{i \mid a_{h i}>0\right\}$.

Proof: Any $\mathbf{t}_{\text {max }} \in \mathbb{T}_{\text {max }, \mathrm{RW}}$ is such that $\mathbf{A t}_{\text {max }} \geq \mathbf{b}, \forall \mathbf{f} \in \mathrm{RW}$ and $\left\|\mathbf{t}_{\max }\right\|_{\infty}=t_{\max , \mathrm{RW}}^{*}$, i.e., $t_{\max , i} \leq t_{\max , \mathrm{RW}}^{*}$ for all $i$. Assume that $\exists i \in I_{h}^{+}$such that $t_{\max , i}<t_{\max , \mathrm{RW}}^{*}$, then:

$$
\mathbf{a}_{h} \mathbf{t}_{\max }=\sum_{a_{h i}>0} a_{h i} t_{\max , i}<t_{\max , \mathrm{RW}}^{*} \sum_{a_{h i}>0} a_{h i}=\max _{\mathbf{f} \in \mathrm{RW}} b_{h}
$$

where the last equality holds since $t_{\max , \mathrm{RW}}^{*}=\frac{\max _{\mathbf{f} \in \mathrm{RW}} b_{h}}{\sum_{a_{h i}>0} a_{h i}}$. This contradicts the fact that $\mathbf{A t}_{\max } \geq \mathbf{b}, \forall \mathbf{f} \in \mathrm{RW}$ so that $t_{\max , i}=$ $t_{\text {max,RW }}^{*}$ for all $i \in I_{h}^{+}$.

Now, let us prove that the set $\mathbb{T}_{\max , R W}$ is a convex polytope since it is the solution set of the following linear inequality system:

$$
\begin{gathered}
t_{\mathrm{max}, i}=t_{\max , \mathrm{RW}}^{*}, \forall i \in I_{h}^{+} \\
t_{\mathrm{max}, i} \leq t_{\max , \mathrm{RW}}^{*}, \forall i \notin I_{h}^{+} \\
\mathbf{A t}_{\max } \geq \mathbf{b}_{\max }
\end{gathered}
$$

where $h$ and $\mathbf{t}_{\max , \mathrm{RW}}^{*}$ are defined in (74) and (24), respectively, and the components of $\mathbf{b}_{\max }$ are defined as $b_{\max , j}=\max _{\mathbf{f} \in \mathrm{RW}} b_{j}$.

Proof: On the one hand, if $\mathbf{t}_{\max }$ verifies (78), (79) and (80), its infinity norm is equal to $t_{\text {max, RW }}^{*}$ and $\mathbf{A t}_{\text {max }} \geq \mathbf{b}, \forall \mathbf{f} \in \mathrm{RW}$ by definition of $\mathbf{b}_{\max }$. Hence, $\mathbf{t}_{\max }$ belongs to $\mathbb{T}_{\max , \mathrm{RW}}$. On the other hand, if $\mathbf{t}_{\max } \in \mathbb{T}_{\text {max,RW }}$ then (79) is satisfied since $\left\|\mathbf{t}_{\text {max }}\right\|_{\infty}=t_{\text {max }, \mathrm{RW}}^{*},(80)$ is true since $\mathbf{A \mathbf { t } _ { \operatorname { m a x } }} \geq \mathbf{b}, \forall \mathbf{f} \in \mathrm{RW}$, and (78) is true according to (76).

\section{APPENDIX $\mathrm{H}$}

\section{CAlculation of $\mathbf{t}_{\text {MAX,RW }}^{m}$ AND Algorithm 2}

In addition to being the solution set of (78)-(80), $\mathbb{T}_{\max , \mathrm{RW}}$ is also the solution set of:

$$
\begin{gathered}
t_{\max , i}=t_{\max , \mathrm{RW}}^{*}, \forall i \in I_{h}^{+} \\
t_{\max , i} \leq t_{\max , \mathrm{RW}}^{*}, \forall i \notin I_{h}^{+} \\
\mathbf{A}^{r} \mathbf{t}_{\max }^{r} \geq \mathbf{b}_{\max }^{r}
\end{gathered}
$$

where $\mathbf{t}_{\max }^{r}$ is obtained from $\mathbf{t}_{\max }$ by removing all components of index $i \in I_{h}^{+}, \mathbf{A}^{r}$ is obtained from $\mathbf{A}$ by removing all columns of indices $i \in I_{h}^{+}$and the components $b_{\max , j}^{r}$ of $\mathbf{b}_{\max }^{r}$ are defined as $b_{\max , j}^{r}=b_{\max , j}-\sum_{i \in I_{h}^{+}} a_{j i} t_{\max , \mathrm{RW}}^{*}$. The proof of this result is straightforward and the same as the one for (58)-(60) in Appendix D. In fact, (83) is simply a rewriting of (80).

Algorithm 2 in Section IV-A, which computes $\mathbf{t}_{\max , \mathrm{RW}}^{m}$, consists in repeatedly solving the minimization problem:

$$
\min _{\mathbf{t}_{\max }^{r}}\left\|\mathbf{t}_{\max }^{r}\right\|_{\infty} \quad \text { subject to } \quad \mathbf{A}^{r} \mathbf{t}_{\max }^{r} \geq \mathbf{b}_{\max }^{r}
$$

where at each step the components $t_{\max , R W, i}$ of $\mathbf{t}_{\max , \mathrm{RW}}^{m}$ corresponding to the indices in $I_{h^{r}}^{+}=\left\{i \mid a_{h^{r}}>0\right\}$ are computed. Following the same methodology and arguments used in Appendix D to prove the validity of Algorithm 1, it can be proved that $\mathbf{t}_{\max , \mathrm{RW}}^{m}$ computed in Algorithm 2 belongs to $\mathbb{T}_{\text {max }, \mathrm{RW}}$, that its components $t_{\text {max }, i}^{m}$, successively computed in while loop in Algorithm 2, are smaller and smaller, and that Algorithm 2 terminates in at most $m$ steps. A more direct proof of the validity of Algorithm 2 can be obtained by noticing that:

$$
\mathbf{A t}_{\max } \geq \mathbf{b}, \forall \mathbf{f} \in \mathrm{RW} \quad \Longleftrightarrow \quad \mathbf{A t}_{\max } \geq \mathbf{b}_{\max }
$$

which holds by definition of $\mathbf{b}_{\max }$ (cf. in Appendix $G$ for this definition). Hence, finding the optimal solution $\mathbf{t}_{\max , \mathrm{RW}}^{m}$ to (23) is the same as computing $\mathbf{t}_{\max }^{m}$ for (13) but with $\mathbf{b}_{\max }$ in place of $\mathbf{b}$ in the inequality constraints $\mathbf{A t}_{\max } \geq \mathbf{b}$.

\section{APPENDIX I}

\section{FIRST RW CASE - PROOF OF (36)}

The RW defined in (35), $\mathrm{RW}_{\text {square, is a convex }}$ polytope whose eight vertices have the following form $\mathbf{f}_{i}=\left[0,0, f_{z}, \pm f_{z} s, \pm f_{z} s, 0\right]^{T}$ with $f_{z}=m_{\min } g$ or $f_{z}=m_{\max } g$. From the general result stated in Section IV-B, $\max _{\mathbf{f} \in \mathrm{RW}_{\text {square }}} b_{j}$ is obtained at the vertex $\mathbf{f}_{i}$ giving the largest value of the linear form $\mathbf{c}_{j} \mathbf{f}$. For all the vertices $\mathbf{f}_{i}$ of $\mathrm{RW}_{\text {square }}, \mathbf{c}_{j} \mathbf{f}_{i}$ has the following form $\mathbf{c}_{j} \mathbf{f}_{i}=f_{z}\left(c_{j f z} \pm s c_{j t x} \pm s c_{j t y}\right)$ where $f_{z}=m_{\min } g$ or $f_{z}=m_{\max } g$. In both cases, $f_{z}>0$ and, hence, $\mathbf{c}_{j} \mathbf{f}_{i}$ is maximized at a vertex $\mathbf{f}_{i}$ such that:

$$
\mathbf{c}_{j} \mathbf{f}_{i}=f_{z} \mu \text { with } \mu=c_{j f z}+s\left(\left|c_{j t x}\right|+\left|c_{j t y}\right|\right) .
$$

Finally, according to (86), to maximize $\mathbf{c}_{j} \mathbf{f}_{i}, f_{z}$ is given by:

$$
f_{z}= \begin{cases}m_{\max } g & \text { if } \mu \geq 0 \\ m_{\min } g & \text { if } \mu<0\end{cases}
$$


so that the components of the vertex $\mathbf{f}_{i}$ of $\mathrm{RW}_{\text {square }}$ yielding the maximum of $\mathbf{c}_{j} \mathbf{f}$ (and thus the maximum of $b_{j}$ ), are:

$$
\begin{aligned}
& f_{x}=f_{y}=t_{z}=0 \\
& f_{z}=\left\{\begin{array}{lll}
m_{\max } g & \text { if } & c_{j f z}+s\left(\left|c_{j t x}\right|+\left|c_{j t y}\right|\right) \geq 0 \\
m_{\min } g & \text { if } & c_{j f z}+s\left(\left|c_{j t x}\right|+\left|c_{j t y}\right|\right)<0
\end{array}\right. \\
& t_{x}=\operatorname{sgn}\left(c_{j t x}\right) f_{z} s, \quad t_{y}=\operatorname{sgn}\left(c_{j t y}\right) f_{z} s .
\end{aligned}
$$

\section{APPENDIX J}

\section{THIRD RW CASE - PROOF OF (40)}

Eq. (39) defines a disc in the wrench space and is thus a particular case of an ellipsoid. Eq. (40) can thus be proved by using the result presented in Section IV-C. In brief, for a given force $F_{d}$, this disc is the set of forces $\left(f_{x}, f_{y}\right)$ such that:

$$
f_{x}^{2}+f_{y}^{2} \leq F_{d}^{2} \Longleftrightarrow\left[f_{x}, f_{y}\right] \mathbf{E}\left[f_{x}, f_{y}\right]^{T} \leq 1, \mathbf{E}=\frac{1}{F_{d}^{2}} \mathbf{I}_{2}
$$

where $\mathbf{I}_{2}$ is the $2 \times 2$ identity matrix. From (28) with $\mathbf{e}=0$ and $\mathbf{c}_{j}$ reduced to $\left[c_{j f x}, c_{j f y}\right]$, the forces $f_{x}$ and $f_{y}$ maximizing $\mathbf{c}_{j} \mathbf{f}$ are given by:

$$
\left[\begin{array}{c}
f_{x} \\
f_{y}
\end{array}\right]=\frac{F_{d}^{2}\left[c_{j f x}, c_{j f y}\right]}{F_{d} \sqrt{c_{j f x}^{2}+c_{j f y}^{2}}}=\frac{F_{d}}{\sqrt{c_{j f x}^{2}+c_{j f y}^{2}}}\left[\begin{array}{l}
c_{j f x} \\
c_{j f y}
\end{array}\right] .
$$

Note that, if the line of action of the lateral $F_{d}$ does not pass through $P$, a moment $t_{z}$ around the vertical axis must be balanced. If $r$ denotes the maximum distance between the line of action and $P, \mathrm{RW}_{\text {distb }}$ can be redefined as:

$$
\mathrm{RW}_{\mathrm{distb}}=\left\{\mathbf{f}\left|\sqrt{f_{x}^{2}+f_{y}^{2}} \leq F_{d},\right| t_{z} \mid \leq F_{d} r, f_{z}=t_{x}=t_{y}=0\right\}
$$

and the wrench yielding the maximum of $\mathbf{c}_{j} \mathbf{f}$ has $f_{x}$ and $f_{y}$ given in (90) and $t_{z}=\operatorname{sgn}\left(c_{j t z}\right) F_{d} r$.

\section{APPENDIX K \\ TESTING SENSITIVITY TO DIMENSIONAL PARAMETER UNCERTAINTIES}

The method used to test sensitivity to dimensional parameter uncertainties consists in computing the maximum value of the smallest maximum cable tension $t_{\text {max. W }}^{*}$ considering that the CDPR drawing and attachment points can be located anywhere in given (tolerance) cubes centered at their nominal values. This computation is achieved by numerically solving the following optimization problem:

$$
\max _{\mathbf{x}} \quad t_{\max , \mathbb{W}}^{*} \quad \text { subject to } \quad \mathbf{x}^{m} \leq \mathbf{x} \leq \mathbf{x}^{M}
$$

where the $(6 m)$-dimensional vectors $\mathbf{x}, \mathbf{x}^{m}$ and $\mathbf{x}^{M}$ contain the coordinates of all the CDPR attachment and drawing points and the lower and upper bounds on these coordinates, respectively. $\mathbf{x}^{m}$ and $\mathbf{x}^{M}$ can be directly obtained from the tolerance cube definitions. The componentwise inequalities $\mathbf{x}^{m} \leq \mathbf{x} \leq \mathbf{x}^{M}$ mean that the coordinates of the drawing and attachment points are uncertain and can be located anywhere in the tolerance cubes. In the sensitivity analysis made in the use case of Section $\mathrm{V}$, the nominal values of the drawing and attachment points were those obtained by means of the optimization problem (46) solved in Phase 2. The tolerance cubes representing the uncertainties on the locations of these points were cubes of edge length equal to $2 \mathrm{~cm}$. Note that the configurations resulting from the optimization (46) made in Phase 2, may have to be reordered according to the values of $t_{\text {max, }}^{*}$ obtained by solving (92). A configuration was considered to be (too) sensitive to uncertainties when $t_{\max }^{*} \mathbb{W}$ computed by means of (92) shows a strong increase compared to the one computed in (46), e.g., twice as large.

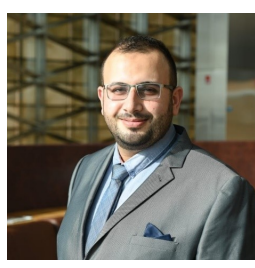

Hussein Hussein received a Ph.D. degree in engineering sciences and microsystems from University of Franche-Comté, France in 2015. From 2017 to 2018, he served as a research scientist at LIRMM, CNRS, University of Montpellier, France. Since January 2019, he is a post-doctoral researcher at King Abdullah University of Science and Technology, KAUST. His current research is focusing on the development of robotic systems and the modeling, design and fabrication of microsystems.

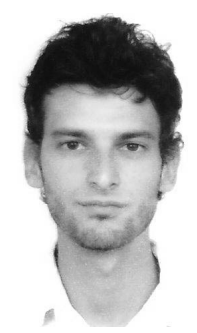

João Cavalcanti Santos received B.Eng. and M.Sc. degrees in mechanical engineering from the University of São Paulo, São Carlos, Brazil in 2015 and 2017, respectively. He received a Ph.D. degree from the LIRMM, Montpellier, France, in 2020, working in the European H2020 project Hephaestus. He is currently a Postdoctoral Fellow with INSERM, Montpellier, France. His main research interests are robot design and control, numerical optimization and model predictive control.

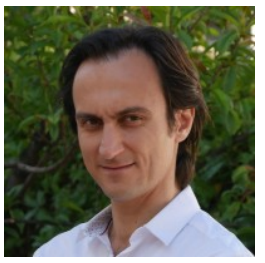

Jean-Baptiste Izard received an M.Sc. in mechanical engineering from Institut Suprieur de l'Aronautique et de l'Espace - SUPAERO (Toulouse, France) in 2006 and his Ph.D. from the Tampere University of Technology (Tampere, Finland) in 2013, in Remote handling and Robotics, received for the work performed at CEA-LIST in Fontenayaux-Roses, France from 2007 to 2010. From 2010 to 2020 he worked for Tecnalia as a research engineer in robotics in Montpellier, France, where he has been in charge of kinematic and detailed design of numerous prototypes, in particular cable-driven parallel robots. He currently works as free-lance mechanical engineer and researcher for Alted in Montpellier, France.

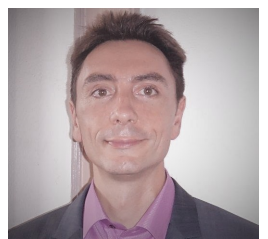

Marc Gouttefarde (M'06) received the B.Eng. degree in mechatronics from Institut National des Sciences Appliques, Strasbourg, France, in 2001, and the M.Sc. and Ph.D. degrees in mechanical engineering from Laval University, Qubec, Canada, in 2002 and 2005, respectively. From 2005 to 2007 , he was a Postdoctoral Fellow with INRIA, SophiaAntipolis, France. He is currently a CNRS senior researcher with LIRMM, Montpellier, France, where he is mainly working on the design, control, and applications of cable-driven parallel robots. Dr. Gouttefarde is the head of LIRMM robotics department and associate editor of the ASME Journal of Mechanisms and Robotics (JMR). 